\title{
Logic Circuits Based on Molecular Spider Systems
}

\author{
Dandan Mo ${ }^{\mathrm{a}}$, Matthew R. Lakin ${ }^{\mathrm{a}, \mathrm{b}, \mathrm{c}}$, Darko Stefanovic ${ }^{\mathrm{a}, \mathrm{c}, *}$ \\ ${ }^{a}$ Department of Computer Science, University of New Mexico \\ ${ }^{b}$ Department of Chemical and Biological Engineering, University of New Mexico \\ ${ }^{c}$ Center for Biomedical Engineering, University of New Mexico
}

\begin{abstract}
Spatial locality brings the advantages of computation speed-up and sequence reuse to molecular computing. In particular, molecular walkers that undergo localized reactions are of interest for implementing logic computations at the nanoscale. We use molecular spider walkers to implement logic circuits. We develop an extended multi-spider model with a dynamic environment wherein signal transmission is triggered via localized reactions, and use this model to implement three basic gates (AND, OR, NOT) and a cascading mechanism. We develop an algorithm to automatically generate the layout of the circuit. We use a kinetic Monte Carlo algorithm to simulate circuit computations, and we analyze circuit complexity: our design scales linearly with formula size and has a logarithmic time complexity.

Keywords: molecular spiders; logic circuits; parallel evaluation; localized signal transmission.
\end{abstract}

\section{Introduction}

Molecular walkers are synthetic molecular machines inspired by natural biological motors. Previous studies [1-10] have shown that walkers can move directionally and autonomously on a pre-programmed track via localized reactions. Spatial locality can meet the challenges of computation speed-up and sequence reuse that are faced in molecular computing when all the reactants diffuse freely in a well-mixed solution [11, [12]. Hence, a walker system with inherent spatial locality has potential to perform more

\footnotetext{
* Corresponding author

Email address: darko@cs.unm.edu (Darko Stefanovic)
}

Preprint submitted to BioSystems

February 6, 2016 
complex computational tasks. We investigate the computational power of a walker system by using it to implement scalable logic circuits.

We consider a molecular spider system, where a spider is a type of multi-legged molecular walker. A molecular spider [13], with a rigid body and several flexible legs, moves stochastically on a surface formed by sites containing DNA segments, and can exhibit biased behavior owing to different reactions with fresh sites (catalytic cleavage) and visited sites (dissociation). We extend previous models [3, 14-17] to implement three basic logic gates (AND, OR, NOT), and cascade the gates to construct logic circuits. Unlike previous models where molecular spiders exhibit biased behaviors, our model uses multiple spiders that are assumed to behave unbiasedly with equal transition rates to all reachable sites. Sites are divided into normal sites, which are immutable, and functional sites, which can be altered via catalytic cleavage and/or strand displacement. We can encode signals into functional sites. Signal transmission $[11,18]$ is triggered locally when a spider interacts with a signal-carrying site, which dynamically changes the state of the spider or of the environment. We call this extended system an active molecular spider system.

In our design, each variable is represented by a moving spider with two legs and one arm. The arm has two possible states, 0 or 1 , representing the Boolean value of the signal the spider carries. Each gate is represented by a layout of different sites on a 2D lattice. In a single gate, spiders with different values will take different paths from their input locations. We arrange different functional sites along different paths, such that only a spider with the correct computation result will be directed to the output location via interactions between spiders and functional sites. On reaching the output location, a spider reports the computation result, and we call it the reporting spider. We cascade logic gates by connecting them such that only the reporting spider leaves the upstream gate and enters the downstream gate. We design a mechanism for exit from gates to implement gate cascades that allow parallel evaluation. As an example, 35 Figure 5 will show a logic circuit where input spiders $X$ and $Y$ are initially placed at the input locations of two NOT gates, and the NOT gates are connected to the same AND gate via exit mechanisms. Spiders move within the circuit, and the spider reaching the final output location reports the computation result. 
There has been much previous work on molecular logic circuits using different in a well-mixed solution use a high and a low relative concentration of a species to represent Boolean values 1 and 0 , or they use two separate species in a dual-rail encoding. In deoxyribozyme-based circuits [22-26], substrates are cleaved by active DNA enzymes if they bind together. Catalytic cleavage between a substrate and a DNA enthe environment. This change is detectable, and the detection of a fluorescence change represents Boolean value 1 . Here, we use spiders with arm state 1 or 0 to represent Boolean values, which removes potential ambiguity from result reporting because we do not need to decide whether a concentration is high or low, or whether there indeed exists a fluorescence change.

Using an extended active multi-spider system, while keeping the advantages related to spatial locality, our design ensures modularity, unambiguity, and scalability. We will describe the model in Section 2 , and show how to construct logic circuits in Section 3 , with simulation results. A formal definition of the model is given in Section 4 We

\section{Model Description}

Our long-term goal is to realize the circuits we describe here with a physical implementation based on molecular spiders [2, 13]. Therefore, our model draws from the existing models of molecular spiders [3, 4] and extends them to describe the richer functionalities of the walkers we hope to build. In spite of these extensions, we will use the evocative term "spider" throughout the paper.

A molecular spider has a body and three limbs, two legs and an "arm", which it can use to attach to chemical sites on a surface. There is exclusion: at most one limb can 
be attached to a given site at a time. Different types of sites are laid out on a square lattice, $\mathbb{Z}^{2}$. A set of contiguous sites can form a track on which the spiders can move.

We model a spider's body as a single point, and the limbs as having equal length. This leads to the following postulated "hand-over-hand" gait [4]: at any given time, exactly two limbs are attached to the surface, and they are attached to nearest-neighbor sites. We call the sites a limb has bound to the attachment points. When a spider limb leaves a site, we assume it can quickly reattach to the surface, so there are always two attachment points for each spider, and they are adjacent to each other. A transition step occurs when a spider detaches one of its limbs from an attachment point $p \in \mathbb{Z}^{2}$, and attaches to a site $p^{\prime} \in \mathbb{Z}^{2}$. Figure 1 shows a transition step of a spider where there are four reachable sites that the limb can potentially transit to. However, a limb might not attach to a reachable site because whether a reachable site is available depends on the state of the site and of the limb, which will be discussed in Section 3 and Section 4 . When multiple spiders are moving on the track, one spider cannot attach to a site occupied by another spider.

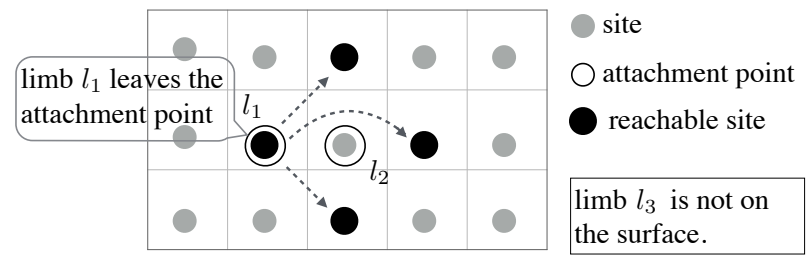

Figure 1: A spider has $\operatorname{limb} l_{1}$ and $\operatorname{limb} l_{2}$ attached to the surface. When limb $l_{1}$ detaches from the left attachment point, four sites represented by the black dots are reachable for limbs $l_{1}$ and $l_{3}$. The arrows show the transitions of a spider to other sites via hand-over-hand movement.

Spiders move stochastically on the track, interacting with the normal sites. If they attach to functional sites, signal transmission is triggered locally between two adjacent sites, or between a site and the spider attached to it. Changes to the sites and spiders may happen during a step, which is crucial in the operation of a logic circuit. In the next section, we will explain how to use different sites to construct three basic logic gates (AND, OR and NOT, thus complete for Boolean logic), and to cascade them to construct a logic circuit. 
Each spider represents a Boolean variable. The value of the spider is indicated by its arm state, which is either 0 or 1 . A logic circuit is formed by cascades comprising the basic logic gates. A logic gate is implemented as an arrangement of different sites on a square lattice, including an output location and input locations. When spiders begin moving from the input locations, their interactions with the sites lead to changes to the sites and the spider values, which ends with one spider reaching the output location, and the value of this spider represents the computation result of the logic circuit.

\subsection{Normal Sites and Functional Sites}

We define the set of site types as $S=S_{\text {norm }} \cup S_{\text {fun }}$, where the normal sites $S_{\text {norm }}=$ $\left\{s_{l}, s_{1}, s_{0}\right\}$ are non-alterable and the functional sites in $S_{f u n}$ are alterable. A normal site of type $s_{l}$ binds to a spider's leg, and is used for the "wires" of a logic circuit. Sites of type $s_{0}$ and $s_{1}$ bind to the spider's arm if it has type 0 or 1 , respectively. Sites of type $s_{0}$ and $s_{1}$ are placed at the beginning of two separate paths that branch out from a junction, directing a spider with different values to different paths (Figure2).

The junction design is used in the constructions for all gate types. Each logic gate has a set of functional sites placed on the paths branching out from the junction. After the spiders take their own paths at the junction according to their values, they will encounter different functional sites. The interactions between the spiders and the functional sites cause changes to the spider and the sites, directing one spider to the output location, to report the result of the gate computation.

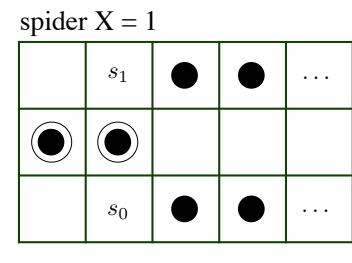

normal site $s_{l}$
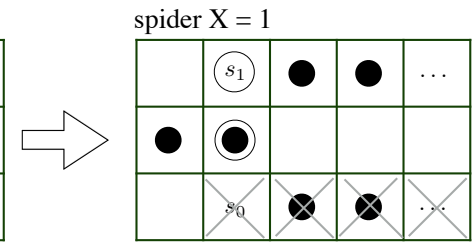

$\bigcirc$ attachment point

Figure 2: If a spider has an arm type of 1 , it binds to site $s_{1}$ at a junction. If a spider has an arm type of 0 , it binds to site $s_{0}$ at a junction. Here a spider $X=1$ follows the upper path by attaching to site $s_{1}$. It cannot follow the lower path. 
Before going into the details of each gate, we first introduce some important features of functional sites. (1) A functional site has a state among \{on, off, trapped\}. The spider can bind to an "on"-state site, cannot bind to an "off"-state site, and cannot leave a "trapped"-state site by itself. (2) A functional site may or may not trap a spider. When it traps a spider, the site's state becomes "trapped". (3) A functional site may contain a signal of "turning on/off" or "switching to 1/0". The signal held in a functional site is sent out once it is attached by a spider. When a spider attaches to a site holding a signal, the signal "turning on/off" is sent to another site, setting its state "on" or "off"; the signal "switching to $1 / 0$ " is sent to the spider, changing its value to 1 or 0 . When a functional site sends out its signal, it has no signal remaining. Signal transmission is allowed between a site and a spider that is attached to the site, or between two sites that are adjacent to each other. These features could be implemented via DNA strand displacement as we shall discuss further in Section 7. We will discuss the AND and OR gate designs in Section 3.2 and the NOT gate design in Section 3.3.

\subsection{Designs of the AND and OR Gates}

We use three types of functional sites $s_{t}, s_{p}$, and $s_{u}$ in the designs of the AND and OR gates. Site $s_{t}$ can trap the spider attaching to it, so we place a site $s_{t}$ at the output location of the gate. The AND gate and OR gate each have two input spiders initially located at the two input locations, which are two junctions as shown in Figure 2 Each input spider selects one of two possible paths when computation begins, where one path leads to the output location without any functional sites and the other path is merged into a crossroads in the middle of the lattice. We place an initially "off"-state site $s_{p}$ at the heart of the crossroads, which blocks the central path from the crossroads to the output location. We place a site $s_{u}$ adjacent to site $s_{p}$, which will send a "turning-on" signal to unblock site $s_{p}$ when a spider attaches to it, and trap that spider at the same time. The cooperation between sites $s_{u}$ and $s_{p}$ guarantees that only when both spiders meet at the crossroads can a spider take the central path to the output location.

Figure 3 shows the layout of the AND gate and OR gate. We explain how the AND gate works under four possible input assignments, and the OR gate follows a similar 
junctions as their input locations. When spiders $X$ and $Y$ are both 0 , they both take the path starting with site $s_{0}$, which leads to the output location without any functional sites. In this case, whichever spider reaches the output location will have value 0 . The reporting spider reports that the result of $0 \wedge 0$ is 0 . When spider $X=0$ and spider

$Y=1$, spider $Y$ takes the path starting with site $s_{1}$, and becomes stuck at the crossroads because site $s_{p}$ is "off". Spider $X$ takes the path starting with site $s_{0}$, and will eventually reach the output location, reporting the result of $0 \wedge 1$ is 0 . When spider $X=1$ and spider $Y=0$, spider $X$ gets to the crossroads via the path starting with site $s_{1}$, and is trapped at the crossroads due to the sites $s_{t}$ and $s_{u}$ placed on that path. Spider $Y$ is the only spider that can reach the output location in this case, reporting the result of $1 \wedge 0$ is 0 . When both spiders are 1 , they meet at the crossroads. Site $s_{p}$ is turned on by the signal sent from site $s_{u}$, so spider $Y$ can take the central path leading to the output location. Since spider $X$ is trapped at the crossroads, only spider $Y$ can reach the output location, reporting the result of $1 \wedge 1$ is 1 .

Following a similar design, the layout of the OR gate is shown in Figure 3 When both spiders are 0 , they meet at the crossroads. Spider $X$ is trapped on sites $s_{t}$ and $s_{u}$, and spider $Y$ takes the unblocked central path to the output location, reporting the result of $0 \vee 0$ is 0 . Under other input assignments, the 0 -valued spider takes the path to the crossroads and becomes stuck there: only the 1-valued spider can reach the output location, reporting the result of $1 \vee 0,0 \vee 1$, and $1 \vee 1$ is 1 .

\subsection{Design of the NOT gate}

We use five types of functional sites in the NOT gate design. As shown in the layout of the NOT gate in Figure 4, site $s_{t}$ which can trap a spider that attaches to it is placed on the output location. Sites $s_{1 \rightarrow 0}, s_{r}^{I}, s_{r}^{I I}$ and sites $s_{0 \rightarrow 1}, s_{r}^{I}, s_{r}^{I I}$ form two different switch mechanisms $S W_{1 \rightarrow 0}$ and $S W_{0 \rightarrow 1}$ that are laid on two separate paths. The NOT gate has one input spider which is initially placed at a junction as the input location. Two separate paths branch out from the junction: one is taken by the 1-valued spider and contains mechanism $S W_{1 \rightarrow 0}$ that can change the spider value to 0 , whereas the other is taken by the 0 -valued spider and contains mechanism $S W_{0 \rightarrow 1}$ that can change the spider value to 1 . When a spider moves through a switch mechanism, its value is switched 


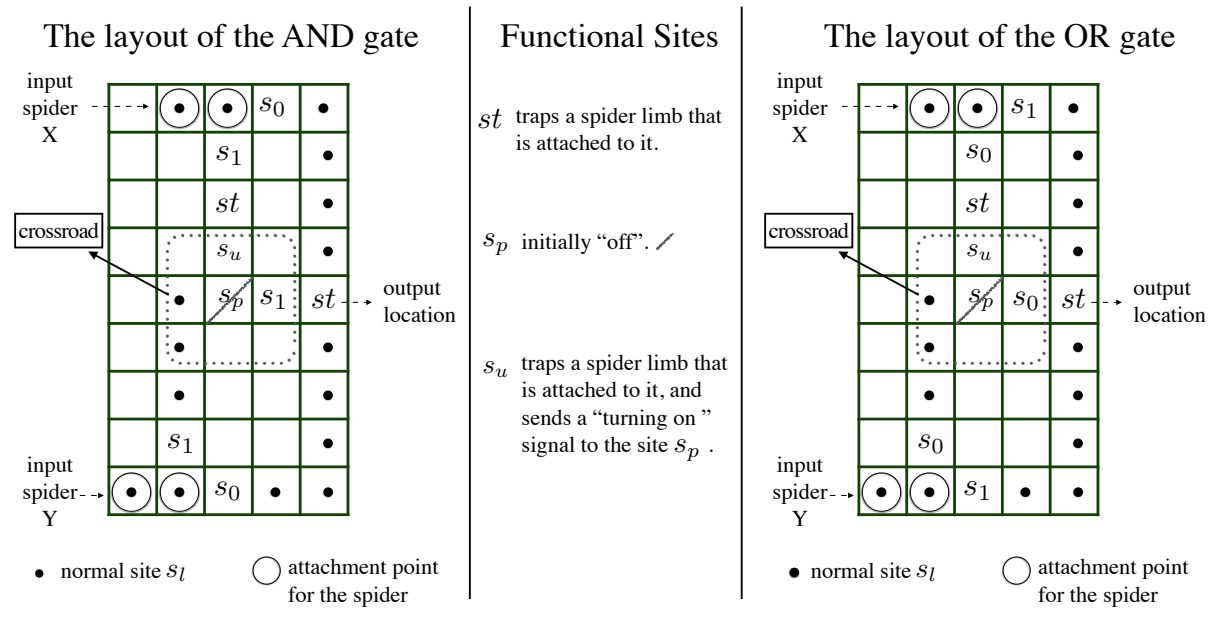

Figure 3: The layout of the AND gate and OR gate. Three functional sites $s_{t}, s_{p}$, and $s_{u}$ used in the designs of these two gates are listed in the middle column. Normal site $s_{1}$ can only bind to an 1-valued spider and normal site $s_{0}$ can only bind to a 0 -valued spider. In the AND gate, when both spiders are 1 , they meet at the crossroads in the middle. Spider $X$ gets trapped at sites $s_{t}$ and $s_{u}$, site $s_{u}$ sends a "turning-on" signal to unblock site $s_{p}$, allowing spider $Y=1$ to take the unblocked central path from site $s_{p}$ to the output location . Under other input assignments, the 1-valued spider gets stuck at the crossroads, so only the 0 -valued spider can reach the output location. Therefore, the AND gate yields 1 when both spiders are assigned 1 , and yields 0 in all other cases. Similarly, in the OR gate, when both spiders are 0 , they meet at the crossroads in the middle and only spider $Y=0$ can reach the output location. Under other input assignments, the 0 -valued spider gets stuck at the crossroads, so only the 1-valued spider can reach the output location. Therefore, the OR gate yields 0 when both spiders are assigned 0 , and yields 1 in all other cases.

and its backward route is cut off. We explain how mechanism $S W_{1 \rightarrow 0}$ works with a 1-valued spider as an example; mechanism $S W_{0 \rightarrow 1}$ works analogously.

Mechanism $S W_{1 \rightarrow 0}$ is formed by three neighboring functional sites along the horizontal direction: $s_{1 \rightarrow 0}, s_{r}^{I}, s_{r}^{I I}$. We use a staging transition diagram in Figure 4 to describe how mechanism $S W_{1 \rightarrow 0}$ changes a 1-valued spider to be 0 , and cuts off the backward route of the spider. A stage transition shows the change of the spider's location, value or the site states. At stage (1), all sites are "on" initially. Site $s_{1 \rightarrow 0}$ can trap a spider, and contains a "switching to 0 " signal that will be sent to its left site when a spider attaches to it. Therefore, when a 1-valued spider attaches to $s_{1 \rightarrow 0}$, it is trapped and receives the signal changing its value to 0 , causing a transition to stage (2). At 


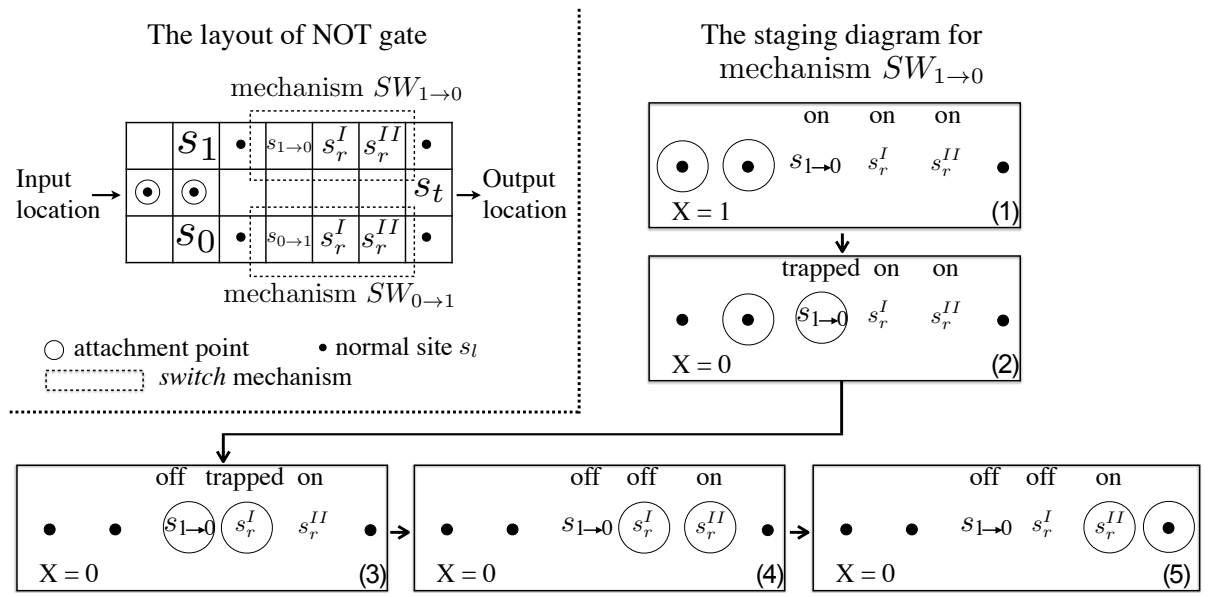

Figure 4: The layout of gate NOT is shown in the figure. The function of mechanism $S W_{1 \rightarrow 0}$ is to switch a spider's value from 1 to 0 and cuts off its backward route. We show how mechanism $S W_{1 \rightarrow 0}$ works in a staging transition diagram, where the spider value is expressed as $X$ and the state of each functional site is shown above it.

stage (2), since the limb trapped at site $s_{1 \rightarrow 0}$ cannot move back, the spider can only move forward by attaching to site $s_{r}^{I}$, which traps the spider and sends out a "turning off" signal to its left site. When site $s_{1 \rightarrow 0}$ receives that signal and turns itself "off", we get to stage (3). At stage (3), the limb trapped on $s_{r}^{I}$ cannot move back, and the spider can only move forward by attaching to site $s_{r}^{I I}$, which sends a "turning off" signal to its left site. When $s_{r}^{I}$ receives that signal and turns itself "off", we get to stage (4). At stage (4), the limb on $s_{r}^{I}$ can transit to a normal site on the right of $s_{r}^{I I}$, while the limb on $s_{r}^{I I}$ cannot move back to $s_{1 \rightarrow 0}$ which is "off". The spider can only move forward to get to stage (5). At stage (5), sites $s_{r}^{I}$ and $s_{r}^{I I}$ are "off", and the spider cannot walk back. When a spider goes through these five stages, its value is switched and its backward route is cut off. The mechanism $S W_{0 \rightarrow 1}$ comprising $s_{0 \rightarrow 1}, s_{r}^{I}, s_{r}^{I I}$ follows similar staging transitions, the only difference being that a 0 -valued spider becomes 1 in the stage transition (1) to (2). 


\subsection{Gate Cascades}

limb transits from one site to another) of each spider is 1 . 


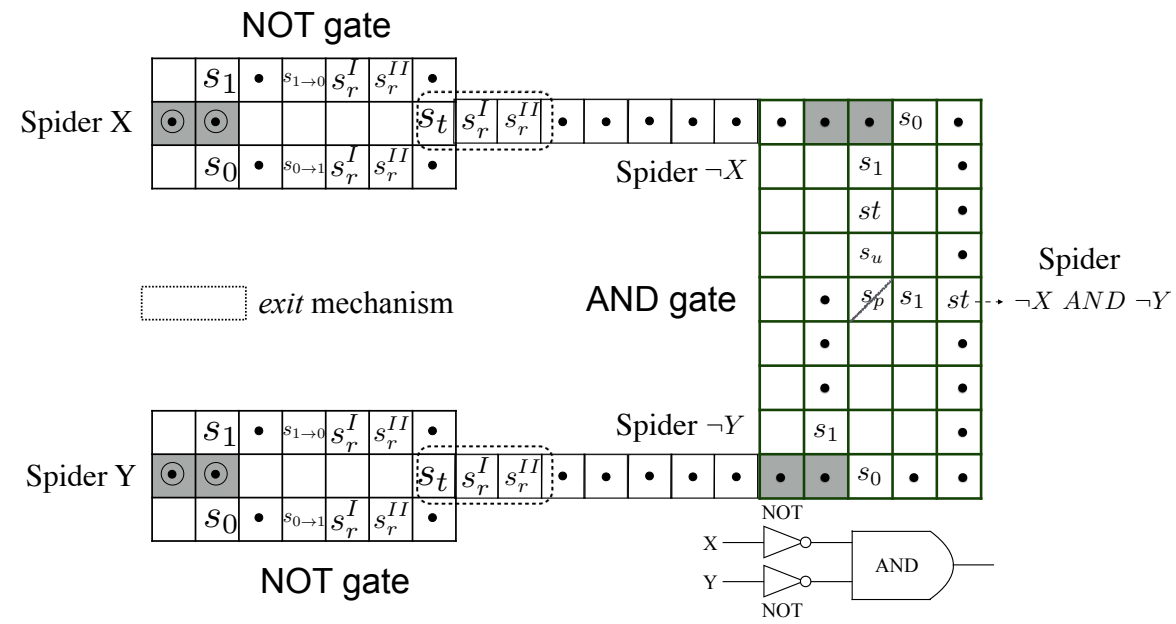

Figure 5: A logic circuit: $(\neg X \wedge \neg Y)$. The input locations of each gate are highlighted in grey. Spiders $X$ and $Y$ exit the NOT gate, becoming spider $\neg X$ and $\neg Y$ after passing through the exit mechanisms. The AND gate computation begins whenever a spider enters the AND gate. The spider reaching the output location of the AND gate represents the computation result $\neg X \wedge \neg Y$.

Simulation results for the three basic gates AND, OR, and NOT are shown in Figure 6. The computation time of a logic circuit $\neg X \wedge \neg Y$ is shown in Figure 6 as well. Under a certain input assignment, the computation time follows a long-tailed distribution because spiders move stochastically. The computation time is the time spent on traversing the path taken by the reporting spider that reaches the output location. In all simulation runs, the output spider produced the correct output value.

\section{Model Definition}

The molecular spider circuit proposed can be modeled as a continuous time Markov model. The environment in this model contains normal sites that are non-alterable and functional sites that are alterable via the interactions between the molecular spiders and the sites. Here we first give the definitions of the site types and transition rules of functional sites, and then the definition of the entire model. 

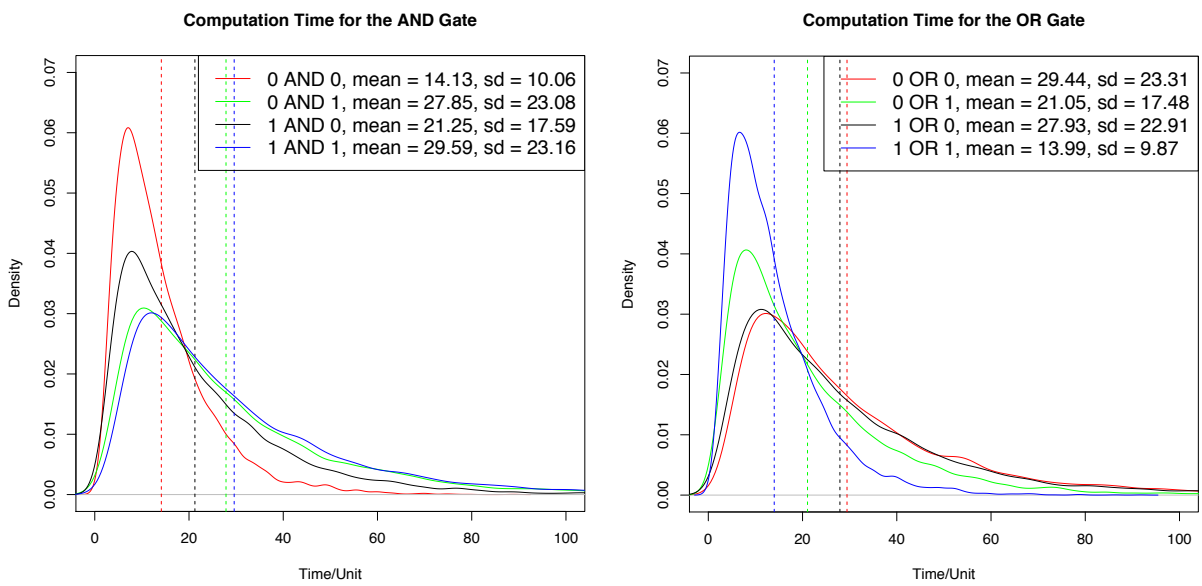

Computation Time for the NOT Gate

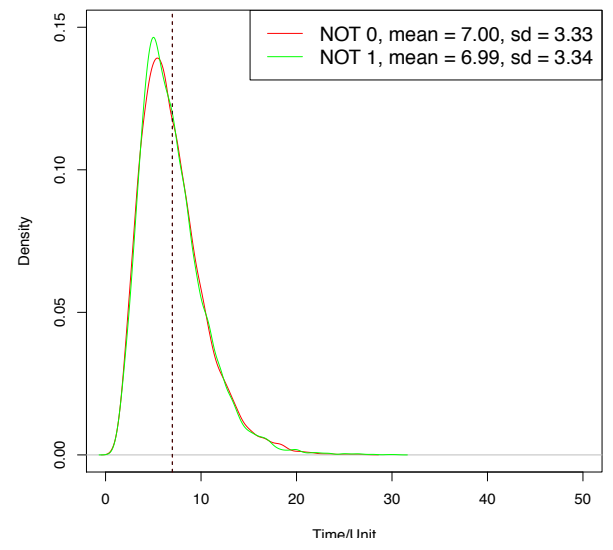

Computation Time for the logic circuit: (NOT X) AND (NOT Y)

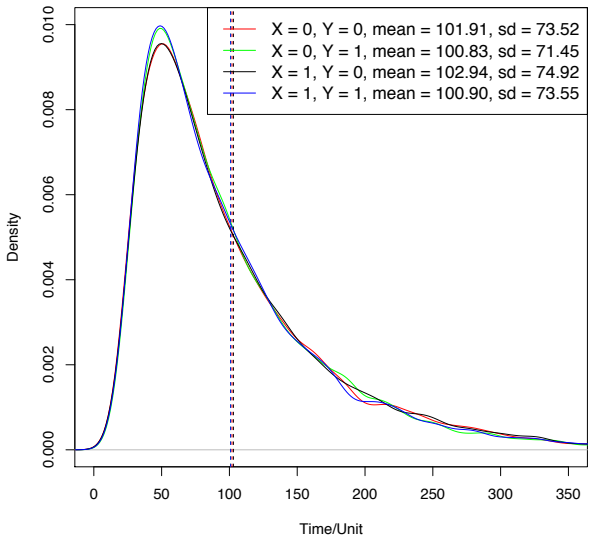

Figure 6: The computation time distributions for gates AND, OR and NOT under four possible input assignments are shown in the figure. Each curve in one gate represents a time distribution under one assignment. The dotted line indicates the mean value of computation time under one assignment in the simulation. The standard deviation for each curve is shown in the legend. The computation time distributions for the logic circuit $(\neg X \wedge \neg Y)$ in Figure 5 under four possible input assignments are shown in the figure. 


\subsection{Site types and transition rules}

Sites are categorized into normal sites and functional sites. A normal site $s \in$ $S_{\text {norm }}=\left\{s_{l}, s_{0}, s_{1}\right\}$ has no state. Site $s_{l}$ binds to the spider's leg. Sites $s_{0}$ and $s_{1}$ bind to the spider's arm if it has type 0 or 1 , respectively.

A functional site $s \in S_{\text {fun }}$ has a state of "on", "off" and "trapped". The site state transition diagram is:

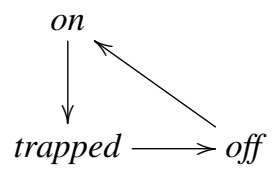

A spider limb can only attach to an "on"-state site. An "off"-state site is non-alterable. The limb trapped on a "trapped"-state site cannot leave the site by itself. Whether a site can trap a spider is indicated by $T R \in\{0,1\}$ : a site with $T R=1$ will trap a spider when a limb attaches to it. A functional site may change the spider's value, or the state of another site, by sending out a signal to the spider or another site. Define

$$
\text { signal }=(\text { val }, d) \text { or null, where } d \in \mathbb{Z}^{2} \text { and val } \in\{\text { on, off, trapped, } 1,0\} .
$$

Suppose a functional site is located at $(x, y)$. If it holds a signal $=\left(v a l, d=\left(d_{x}, d_{y}\right)\right)$ there to val. When $d=(0,0)$, the val field of the signal is either 1 or 0 , which is sent to the spider, setting the spider's value to 1 or 0 .

Therefore, a functional site $s \in S_{\text {fun }}$ can be defined as

$$
s=(\text { state }, T R, \text { signal }) .
$$

The signal held in a site is sent out once a spider limb attaches to the site. When a signal is sent out, the site has no signal remaining, which is express as $s=($ state $, T R, n u l l)$. Once a signal is received by a site or a spider, the site state or the spider's value is changed according to the signal.

In the logic circuit construction, we use two functional sites $s_{u}$ and $s_{p}$ in the AND gate and OR gate, and we design a set of functional sites that form different mecha- 
Table 1: Definitions of different functional sites used in the circuit construction and the transition rules applied to them. Suppose the location of the site is $(x, y)$, define $\left(x^{\prime}, y^{\prime}\right)=\left(x+d_{x}, y+d_{y}\right)$.

\begin{tabular}{l|l|l}
\hline \multicolumn{3}{c}{ Transition Rules } \\
\hline functional site & updated site & other changes \\
\hline$s_{t}=($ on, 1, null $)$ & $s_{t}^{\prime}=($ trapped, 1, null $)$ & \\
\hline$s_{1 \rightarrow 0}=($ on, $1,(0,(0,0)))$ & $s_{1 \rightarrow 0}^{\prime}=($ trapped, 1, null $)$ & $A=0$ \\
\hline$s_{0 \rightarrow 1}=($ on $, 1,(1,(0,0)))$ & $s_{0 \rightarrow 1}^{\prime}=($ trapped, 1, null $)$ & $A=1$ \\
\hline$s_{r}^{I}=($ on, $1,($ off,$d))$ & $s_{r}^{I^{\prime}}=($ trapped, 1, null $)$ & site at $\left(x^{\prime}, y^{\prime}\right)$ turns off \\
\hline$s_{r}^{I I}=($ on $, 0,($ off,$d))$ & $s_{r}^{I I^{\prime}}=($ on, 0, null $)=s_{l}$ & site at $\left(x^{\prime}, y^{\prime}\right)$ turns off \\
\hline$s_{u}=($ on, $0,($ on,$d))$ & $s_{u}^{\prime}=($ on, 0, null $)=s_{l}$ & site at $\left(x^{\prime}, y^{\prime}\right)$ turns on \\
\hline$s_{p}=($ off, 0, null $)$ & $s_{p}^{\prime}=($ on,, 0, null $)=s_{l}$ & \\
& when a “turning-on” sig- & \\
\hline
\end{tabular}

functional sites and the transition rules applied to them. A functional site $s$ transits to site $s^{\prime}$ in the second column, either by receiving a signal or being attached by a spider limb. If $s$ holds a signal, it causes other changes in the last column. In table 1 , the updated sites $s^{\prime}$ in the second column is either a normal site or a trapped site. According to the site state transition diagram, a trapped site can only transit to a "off"-state site that is non-alterable by itself. Since no signals are designed to turn on these "off"-state sites transited from the trapped sites, these "off"-state sites are non-alterable finally. Therefore, all the functional sites in Table 1 are alterable initially and become non-alterable finally. The functional sites used in our design are

$$
\left\{s_{t}, s_{1 \rightarrow 0}, s_{0 \rightarrow 1}, s_{r}^{I}, s_{r}^{I I}, s_{u}, s_{p}\right\},
$$

where each site $s$ among them includes its site transitions under the transition rules described in Table 1. The set of site types is $S=S_{\text {norm }} \cup S_{\text {fun }}$.

A mechanism is a set of neighboring mechanism sites along the same direction. We design three different mechanisms used in the logic circuit construction. The switch mechanism $S W_{1 \rightarrow 0}\left(S W_{0} \rightarrow 1\right)$ contains sites $s_{1 \rightarrow 0}\left(s_{0 \rightarrow 1}\right), s_{r}^{I}, s_{r}^{I I}$, where sites $s_{r}^{I}, s_{r}^{I I}$ con- 
tains the signal of (off, $(-1,0)$ ) which can block its left site. When a spider moves over the switch mechanism, its value is flipped, and its backward route is cut off. The exit mechanism contains sites $s_{t}, s_{r}^{I}, s_{r}^{I I}$. When a spider moves over this mechanism, its backward route is cut off.

When a spider limb leaves a site, this limb can reach 4 sites geometrically (shown in Figure 11. Since sites have different types, whether a site is available for a limb of a spider depends on the spider value and the site types.

Definition 1. Given a spider with value $A$ and a reachable site, we check if the site is available by using the following conditions:

- if the site is occupied by another spider, it is not available.

- else:

1. if the site is a normal site:

(a) if the site is $s_{l}$, it is available;

(b) if the site is $s_{1}$ and $A=1$, it is available;

(c) if the site is $s_{0}$ and $A=0$, it is available;

2. else if the site is a functional site:

(a) if the site is $s_{1 \rightarrow 0}$ and $A=1$, it is available;

(b) if the site is $s_{0 \rightarrow 1}$ and $A=0$, it is available;

(c) if the site is "on"-state, it is available;

3. else, the site is not available.

Using Definition 1, every site is examined among the four reachable sites shown in Figure 1 Those available sites are put into a set $\mathscr{A} \mathscr{V}$.

\subsection{Model definition.}

The active multi-spider system with normal sites and alterable sites can be modeled as a continuous-time Markov process. The state of the model is defined as

$$
X=\left(S_{1}, S_{2}, \ldots, S_{n}, E\right),
$$


where $S_{i}=\left(P_{i}, A_{i}\right)(1 \leq i \leq n)$ describes the state of the $i$-th spider. Set $P_{i}=\left(p_{a}^{i}, p_{b}^{i}\right)$

contains attachment points for the $i$-th spider, and $A_{i} \in\{0,1\}$ represents the Boolean value of the spider. The lattice configuration $E: \mathbb{Z}^{2} \rightarrow S$ shows the layout of different sites, where $S$ is the set of site types. Normal sites can be regarded as having state "on", $T R=0$ and no signal, so the lattice configuration can be redefined as

$$
E: \mathbb{Z}^{2} \rightarrow\{\text { on, off, trapped }\} \times\{1,0\} \times \mathbb{S},
$$

where $\mathbb{S}$ represents the set of signals.

Given a model state $X=\left(S_{1}, S_{2}, \ldots, S_{n}, E\right)$ at time $t$, if a limb leaves an attachment point $p \in P_{i} \in S_{i}$, we use the Definition 1 to obtain a set of available sites $\mathscr{A} \mathscr{V}$. At time $t+\delta$, this limb transits to $p^{\prime} \in \mathscr{A} \mathscr{V}$, changing the set of attachment points to $P_{i}^{\prime}=P_{i}-\{p\} \cup\left\{p^{\prime}\right\}$. The transition rules are used to update $A_{i}$, so that the $i$-the spider state becomes $S_{i}^{\prime}=\left(P_{i}^{\prime}, A_{i}^{\prime}\right)$. The transitions rules also updates $E$, thus the new state is

$$
X^{\prime}=\left(S_{1}, S_{2}, \ldots, S_{i-1}, S_{i}^{\prime}, S_{i+1}, \ldots, S_{n}, E^{\prime}\right) .
$$

\section{Verification}

Verification is a procedure of checking if the given circuit yields the expected results under all possible input assignments. The verification of each single gate (AND, OR, NOT) discussed in previous sections checks if all spiders take the intended paths during the gate computation under all possible input assignments. Assuming all mechanisms work properly in each gate, spiders in each gate always take the intended paths under all input assignments, and only one spider can exit the gate. Therefore, all single gates are verified to work properly under all input assignments. Since our design guarantees modularity, and since all gates are verified to work properly, if all gates in a circuit are cascaded correctly and do not interfere with each other, the circuit is verified to work properly. Therefore, to verify a circuit, it remains to check if the layout of the circuit ensures correct cascading.

\subsection{Geometric Layout Generation}

We develop an algorithm to generate the circuit layout. Given a Boolean function, the algorithm converts the function into a circuit tree and generates a two-dimensional 
layout according to the circuit tree. A Boolean function can be converted into a logic circuit composed of input variables and logic gates of AND, OR, and NOT. In our design, since all gates have one output and at most two inputs, the logic circuit (which is assumed to be feedforward) can be represented by a binary tree. Each tree node represents a gate. A connection between two nodes represents the cascade between two gates, where the output location of the upstream gate (child node) is connected to the input location of the downstream gate (parent node). Our design does not have the fan-out gate that takes one input and outputs multiple copies of the input; instead each variable occurrence is represented by a molecular spider. Using the binary tree representation, the geometric layout problem of a circuit is converted to the problem of constructing a binary circuit tree that represents the given circuit such that gates do not interfere with each other. In the circuit tree construction, the layouts of the tree nodes should not have overlaps, and the layout of a connection between two nodes only joins the layout of the upstream gate at its output location with the layout of the downstream gate at its input location.

Our layout generation algorithm includes two parts: circuit conversion converts a given Boolean function into a circuit tree, whereas circuit tree construction constructs the circuit tree recursively. The circuit tree is a binary Abstract Syntax Tree (AST) representation of the Boolean formula. There are numerous tools that generate the AST for a given Boolean formula, so we only give details of circuit tree construction here, which re-designs all the gates to let them have the same size of geometric layout and constructs the circuit tree by recursively merging subtrees from the leaf nodes to the root node.

\subsection{Redesign of the Gates.}

Figure 7 shows the layouts of single gates of AND, OR, and NOT. Define $E_{g}: \mathbb{Z}^{2} \rightarrow$ $S$ where $S$ is the set of site types and $g \in\{A N D, O R, N O T\}, E_{g}$ is the layout of gate $g$.

A Boolean function can be expressed in a form of Boolean circuit that is composed of AND gates, OR gates, and NOT gates. Since all the gates have only one output location and at most two input locations, assuming each variable occurrence corresponds to a molecular spider, the Boolean circuit can be represented by a binary AST. A tree 
OR gate

\begin{tabular}{|c|c|c|c|c|c|c|c|c|}
\hline$\bullet$ & $\bullet$ & $\bullet$ & $\bullet$ & $s_{t}$ & $\bullet$ & $\bullet$ & $\bullet$ & $\bullet$ \\
\hline$\bullet$ & & & & $\bullet$ & & & & $\bullet$ \\
\hline$s_{1}$ & & & & $s_{0}$ & & & & $s_{1}$ \\
\hline$\odot$ & $s_{0}$ & $s_{t}$ & $s_{u}$ & $s_{p}$ & $\bullet$ & $\bullet$ & $s_{0}$ & $\odot$ \\
\hline$\odot$ & & & & & & & & $\odot$ \\
\hline
\end{tabular}

NOT gate

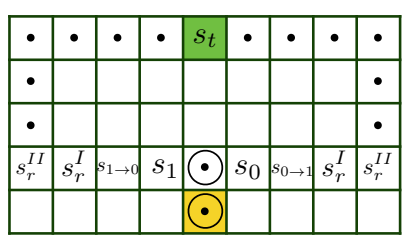

AND gate

\begin{tabular}{|c|c|c|c|c|c|c|c|c|}
\hline$\bullet$ & $\bullet$ & $\bullet$ & $\bullet$ & $s_{t}$ & $\bullet$ & $\bullet$ & $\bullet$ & $\bullet$ \\
\hline$\bullet$ & & & & $\bullet$ & & & & $\bullet$ \\
\hline$s_{0}$ & & & & $s_{1}$ & & & & $s_{0}$ \\
\hline$\odot$ & $s_{1}$ & $s_{t}$ & $s_{u}$ & $s_{p}$ & $\bullet$ & $\bullet$ & $s_{1}$ & $\odot$ \\
\hline$\odot$ & & & & & & & & $\bigodot$ \\
\hline
\end{tabular}

$\bigcirc$ attachment point

- normal site

output location

input location

Figure 7: Geometric layouts of three basic gates with the same size.

node represents a gate, its child nodes represent the upstream gates connected to it, and its parent node is the downstream gate it connects to. For example, a Boolean circuit $\left(x_{1} \vee x_{2}\right) \wedge \neg x_{3}$ can be represented by a binary tree containing three nodes, where the root node is AND gate and it has two child nodes of OR gate and NOT gate. Each node can be mapped to its corresponding layouts shown in Figure 7. The child-parent relation is mapped to a contiguous set of sites connecting an upstream gate and a downstream gate. Therefore, given a Boolean circuit in a binary tree structure, the geometric layout of the circuit is converted to the construction of the tree. Figure 8 shows an example of this conversion procedure.

\subsection{Circuit Tree Construction.}

Figure 7 has provided the layouts of all gates that will be used in the circuit construction. Given a circuit tree, the generation of the circuit layout is to recursively merge the layout of the root node with the layouts of its subtrees. The merging procedure follows a Depth-First-Search (DFS) routine. Leaf nodes representing the input variables do not have layouts. The result of merging a root node (gate) with a leaf node (variable) is the layout of the root node and an additional information indicating the initial position of the variable. For example, circuit $\left(x_{1} \vee x_{2}\right) \wedge \neg x_{3}$ in Figure 8 is the result of merging the layouts of NOT gate (right subtree) and OR gate (left subtree) 


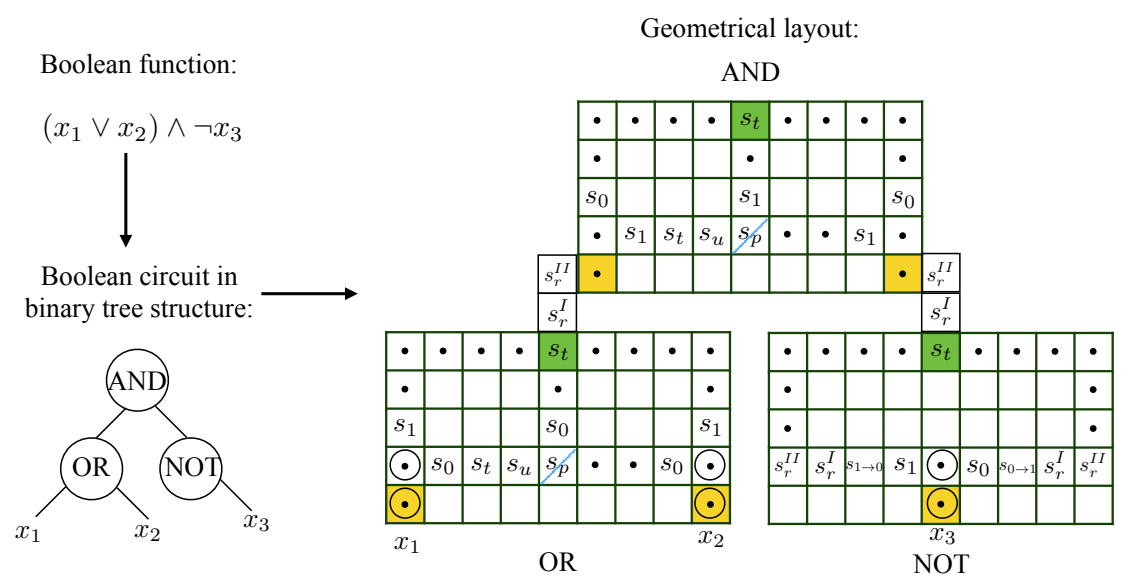

Figure 8: A Boolean function $\left(x_{1} \vee x_{2}\right) \wedge \neg x_{3}$ can be represented by a binary tree. Each tree node is mapped to a gate layout among the layouts predefined in Figure 7 The geometric layout of the circuit can be converted into the construction of a binary tree using the predefined tree nodes.

with the layouts of downstream gate AND (root node). The layout of the merged circuit $\left(x_{1} \vee x_{2}\right) \wedge \neg x_{3}$ includes the cascading paths between gate AND and gates NOT, OR. Initial positions of variables $x_{1}, x_{2}$ and $x_{3}$ are also gained.

We discuss the merging procedure between a root node $(R)$ and its subtrees (left subtree Left and right subtree Right) in two cases.

\subsubsection{Case 1: the root node is a NOT gate}

Suppose the NOT gate only has the right subtree. If Right is a leaf node representing a variable, we place that variable at the input location of the NOT gate and return the layout of the NOT gate. Otherwise we create a new layout $E_{\text {merged }}: \mathbb{Z}^{2} \rightarrow S$ containing the layouts of the root node $E_{R}$ and the right subtree $E_{R i g h t}$. In the merged layout, as shown in Figure 9, the subtree tree is placed below the root node and they keep unit distance to each other vertically. Cascading path between the output location of the subtree and the input location of the root node is constructed using the normal sites. An exit mechanism consisting of sites $s_{t}, s_{r}^{I}$ and $s_{r}^{I I}$ is placed on the cascading path connecting the output location of the subtree circuit and the input location of the root node. 


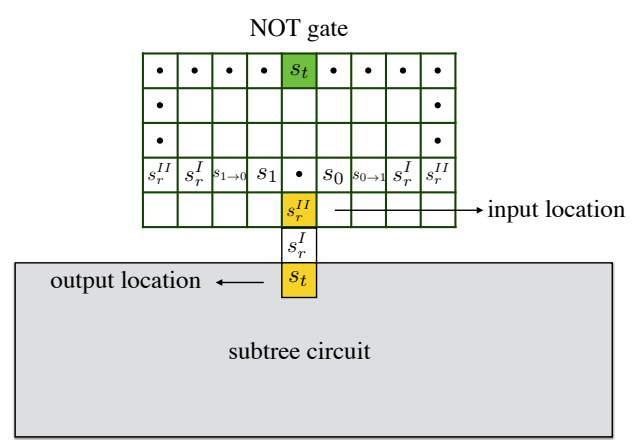

Figure 9: Merging the layout of a NOT gate with its subtree circuit.

\subsubsection{Case 2: the root node is an AND/OR gate}

If two subtrees are both variables, we return the layout of the root node gate and place the variables at the input locations of the root node gate. If one of the subtree circuits is a variable and the other is a circuit, we use the merging procedure shown in Figure 9 to place the subtree circuit below the root node gate and initialize the position of that variable. If both subtrees are circuits, we create a new layout $E_{\text {merged }}: \mathbb{Z}^{2} \rightarrow S$ containing the layouts of the root node $E_{R}$ and the subtrees $E_{L e f t}$ and $E_{R i g h t}$. In the merged layout, the top of the left subtree is aligned horizontally with the top of right subtree. The two subtrees keep unit distance to each other horizontally. The root node gate is placed in the middle between the output locations of subtrees Left and Right. The root node gate keeps unit distance vertically to its subtrees. Figure 10 shows the procedure of merging a root node gate with its two subtree circuits. Two cascading paths containing the exit mechanisms connect the output locations of the subtree circuit and the input locations of the root node gate.

Given a circuit tree that is represented by a binary AST, the procedure of the circuit tree construction ensures that every node in the tree is cascaded correctly with its subtrees. For each node of the tree, its layout and the layouts of its subtrees do not have overlaps, thus all the gates in the tree do not influence with each other. Since all single gates are verified to work properly under all input assignments, and since the circuit layout generation algorithm guarantees the correct cascades between different gates in the circuit, the entire circuit generated using our algorithm is verified to work properly 


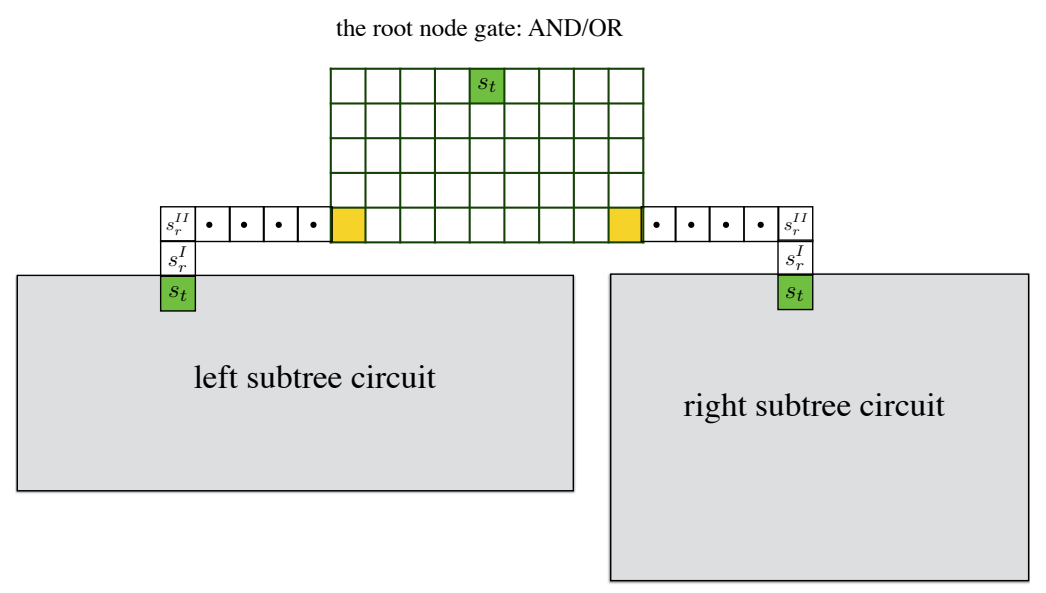

Figure 10: Merging the layout of an AND/OR gate with its subtree circuits.

under all input assignments.

\section{Performance}

The performance of a circuit is usually measured by its computation speed and computation correctness, and the ability to accommodate the increasing size of the circuit (the number of input variables or the circuit depth). In this section, we analyze the complexity of our design and present some simulation results of a small set of circuits.

\subsection{Complexity Analysis}

In a single gate, the computation time $t_{\text {gate }}$ is the traversal time of the spider that reaches the output location. Since the spider moves on the track stochastically, the computation time $t_{\text {gate }}$ is a random variable following a long-tailed distribution, as shown in Figure6,

When a spider leaves a gate or enters a gate, its backward route is cut off due to ${ }^{415}$ the functionality of the exit mechanism, so the computation time of a single gate $t_{\text {gate }}$ can be used to estimate the computation time $t$ of a circuit. For any $n$-variable Boolean function, it can be transformed into 3-CNF, which is a conjunction of $m$ clauses, each a 
disjunction of at most three literals. Since the circuit design allows parallel evaluation, for a clause $m_{i}=\left(l_{1}^{i} \vee l_{2}^{i} \vee l_{3}^{i}\right)$, the computation time of $m_{i}$ is

$$
t_{m_{i}} \leq 2 \times\left(t_{\mathrm{OR}}+t_{\mathrm{NOT}}\right)=O(1) .
$$

Since each clause needs time $t_{m_{i}}$, to evaluate $m$ clauses in parallel, we need $\log m$ AND gate computations that $\operatorname{cost} t_{\mathrm{AND}} \times \log m$, which totally use time

$$
t=t_{\mathrm{AND}} \times \log m+t_{m_{i}}=O(\log m) .
$$

For any Boolean function in 3-CNF with $m$ clauses, there are at most $3 m$ spiders representing the literals. For each clause, at most three NOT gates and two OR gates are needed if all the literals are the negation of a variable, which is a constant number. For $m$ clauses, there needs $m-1$ AND gates. Therefore, the total space complexity is $O(m)$. Hence, the circuit design is scalable because circuit size in the design scales linearly with formula size, and evaluation time is logarithmic in the formula size.

\subsection{Simulation Results}

Here we look at how the computation time varies with the size of the circuit on a set of examples. We investigate a small set of circuits that are in the form of 3-CNF where there are no NOT gates. We first investigate a single clause $\left(x_{1} \vee x_{2} \vee x_{3}\right)$. Computation time distributions of this single-clause circuit under all possible input assignments are shown in Figure 12. The results show that computation time is influenced by the input assignment for a fixed circuit. Different input assignments lead to different computation paths for the input spiders, where each computation path starts from the initial location of the spider and ends at the output location of the circuit. A spider taking the shorter path is more likely to be the reporting spider to reach the output location, which terminates the computation. Therefore, computation time is the time which the reporting spider takes to terminate the computation. Since different input assignments lead to different computation paths of the reporting spider, and different lengths lead to different computation time, computation time for a fixed circuit varies with different input assignments. For the single-clause circuit $\left(x_{1} \vee x_{2} \vee x_{3}\right)$ whose circuit structure is shown in Figure 11, there are two lengths of computation paths of the reporting spider. 
When $x_{3}=1$, spider $x_{3}$ has a shortcut to the output location (indicated by the red-color arrow); otherwise spider $x_{3}$ would wait in the root OR gate until either spider $x_{1}$ or spider $x_{2}$ takes the green path to participate in the root-node gate computation. When $x_{3}=1$, spider $x_{3}$ is more likely to be the reporting spider. The red path taken by spider $x_{3}$ is relatively shorter, so the computation time is relatively shorter. In the results shown in Figure 12, four curves with $x_{3}=1$ are similar to each other, and the mean time and standard deviations of these four curves are smaller compared with other four curves because $x_{3}$ is more likely to be the reporting spider and walks through a shorter path. When $x_{3}=0$, the reporting spider is more likely to take a longer path passing two gates, so the mean computation time and standard deviation are greater, as shown in the curves $000,010,100$, and 110 of Figure 12

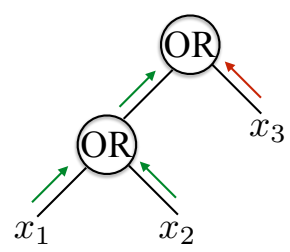

Figure 11: The circuit structure of $\left(x_{1} \vee x_{2} \vee x_{3}\right)$

Assigning 1 to all the variables, we investigate a group of circuits in the form of $3-\mathrm{CNF}$ where the clause number of the circuit is from 1 to 5 . Figure 13 shows the simulation results. We use $(k, h)$ to represent a circuit having $k$ clauses and $h$ heights. Table 2 lists 7 different circuit structures we simulate. The results in Figure 13 show that as the circuit size (the number of clauses) increases, the mean computation time and standard deviation increase. Having fixed the size of the circuit, the mean computation time and standard deviation increase as the circuit height increases, which is reflected in the black and red curves, and the gray and orange curves shown in Figure 13.

The result of complexity analysis shows that our circuit design scales linearly as the circuit size increases. We use simulation to investigate what factors influence the circuit performance, and we find that different input assignments lead to different computation time distributions for a fixed circuit, and the mean computation time and standard deviation increase as the circuit size and circuit height increase when we fix the input 


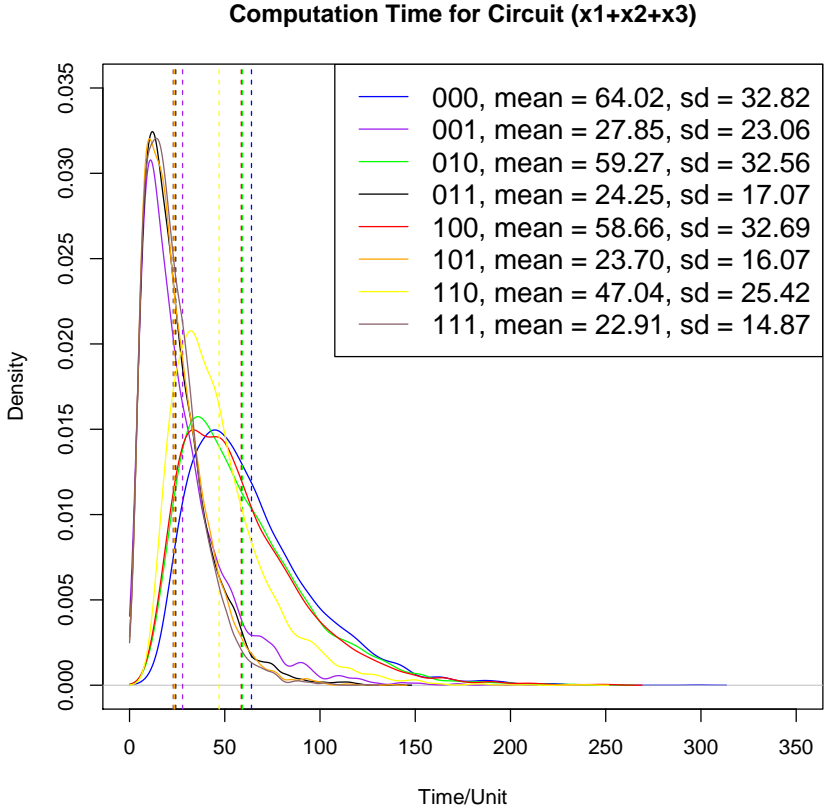

Figure 12: Simulation results of circuit $\left(x_{1} \vee x_{2} \vee x_{3}\right)$ under all input assignments. Each curve represents the computation time of the circuit under one input assignment. Input assignments for variables $x_{1}, x_{2}$ and $x_{3}$ are represented by binary strings as shown in the legend. For example, 101 represents the input assignment $x_{1}=1, x_{2}=0, x_{3}=1$. Dashed lines represent the mean values of computation time. Standard deviations for each curve are shown in the legend. 


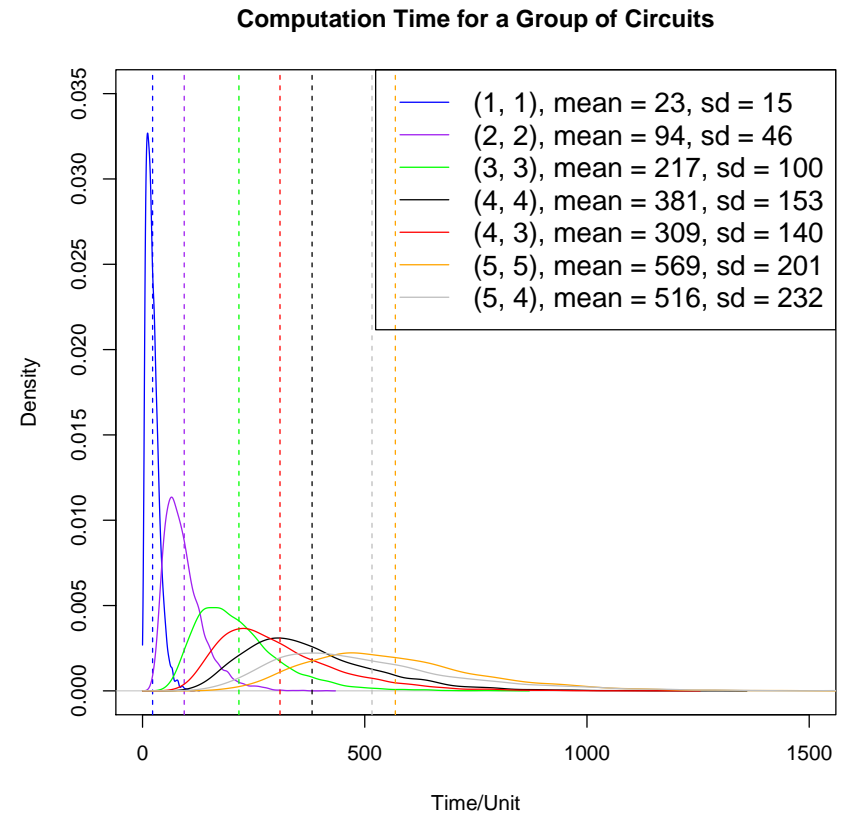

Figure 13: Simulation results of a group of circuits having clause number from 1 to 5 under the input assignments where all variables are 1 . In the legend, each curve $(k, h)$ represents the computation time distribution of a circuit having $k$ clauses and $h$ heights. We show the mean time and standard deviation for each curve in the legend. 
Table 2: A small set of circuits simulated.

\begin{tabular}{l|l|l}
\hline no. & $(k, h)$ & Circuit Structure \\
\hline 1. & $(1,1)$ & $x_{1} \vee x_{2} \vee x_{3}$ \\
\hline 2. & $(2,2)$ & $\left(x_{1} \vee x_{2} \vee x_{3}\right) \wedge\left(x_{1} \vee x_{2} \vee x_{4}\right)$ \\
\hline 3. & $(3,3)$ & $\left(x_{1} \vee x_{2} \vee x_{3}\right) \wedge\left(x_{1} \vee x_{2} \vee x_{4}\right) \wedge\left(x_{1} \vee x_{3} \vee x_{4}\right)$ \\
\hline 4. & $(4,4)$ & $\left(x_{1} \vee x_{2} \vee x_{3}\right) \wedge\left(x_{1} \vee x_{2} \vee x_{4}\right) \wedge\left(x_{1} \vee x_{3} \vee x_{4}\right) \wedge\left(x_{2} \vee x_{3} \vee x_{4}\right)$ \\
\hline 5. & $(4,3)$ & $\left(\left(x_{1} \vee x_{2} \vee x_{3}\right) \wedge\left(x_{1} \vee x_{2} \vee x_{4}\right)\right) \wedge\left(\left(x_{1} \vee x_{3} \vee x_{4}\right) \wedge\left(x_{2} \vee x_{3} \vee x_{4}\right)\right)$ \\
\hline 6. & $(5,5)$ & $\left(x_{1} \vee x_{2} \vee x_{3}\right) \wedge\left(x_{1} \vee x_{2} \vee x_{4}\right) \wedge\left(x_{1} \vee x_{3} \vee x_{4}\right) \wedge\left(x_{2} \vee x_{3} \vee x_{4}\right) \wedge\left(x_{1} \vee\right.$ \\
\hline 7. & $(5,4)$ & $\begin{array}{l}\left(\left(x_{1} \vee x_{2} \vee x_{3}\right) \wedge\left(x_{1} \vee x_{2} \vee x_{4}\right)\right) \wedge\left(\left(x_{1} \vee x_{3} \vee x_{4}\right) \wedge\left(x_{2} \vee x_{3} \vee x_{4}\right) \wedge\left(x_{1} \vee\right.\right. \\
\end{array}$ \\
\hline
\end{tabular}

assignment.

\section{Possible Implementations}

We have sketched two different possible implementations of the model used in the logic circuit construction. We will give details of these two implementations in Section 7.1 and Section 7.2.

\subsection{Possible Implementation I}

We use DNA strand displacement and catalytic cleavage to implement the mechanisms $S W_{1 \rightarrow 0}$ and $S W_{0 \rightarrow 1}$ in a NOT gate and mechanism exit in the gate cascades. Non-alterable sites form the main parts of the track, their structures are single-stranded DNA. Alterable sites form the functional parts of the track, whose structures are either single-stranded DNA with long complementary domains to the spider limbs, or the hairpin structures and bridge structures. We assume that when the binding part between a limb and a site is more than two complementary domains, the bound limb cannot leave that site by itself, and is trapped on that site. 


\subsubsection{Spiders and non-alterable sites.} and 0-typed arm respectively.

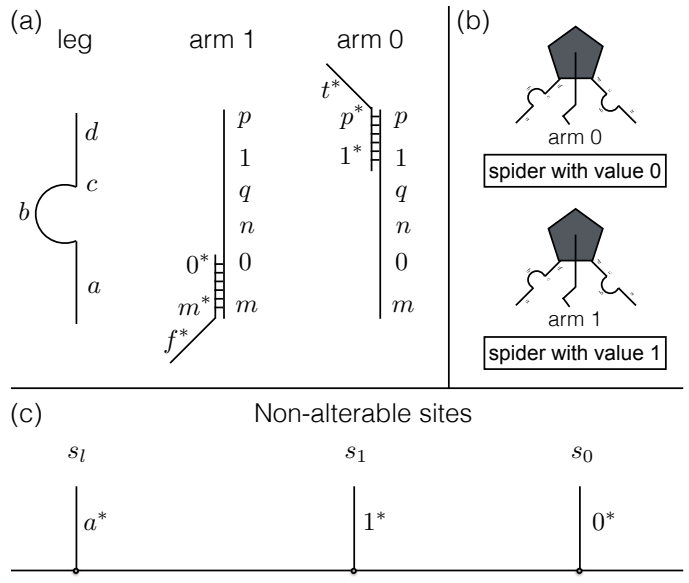

Figure 14: Possible implementation I. The spider structure and non-alterable sites. (a) The leg is a DNAzyme where the domain $b$ catalyzes cleavage of a substrate in mechanisms that will be shown in later figures. The $a$ domain of the leg is complementary to the $a^{*}$ domain of site $s_{l}$, thus the leg can attach to site $s_{l}$. Arm 1 is partially hybridized with an indicator strand $\left(0^{*}, m^{*}, f^{*}\right)$. A spider having an arm of 1 can attach to a site $s_{1}$ since the domain 1 of its arm is complementary to the domain $1^{*}$ of site $s_{1}$. Similarly, a spider having an arm of 0 can attach to a site $s_{0}$. (b) Two types of spiders are shown at top right. Each spider has two legs and an arm. (c) The non-alterable sites are single-stranded DNA with a single domain. They are tethered to the surface of a lattice, where the small gray dots in the figure represent the tethered points. We assume the binding strength between a pair of complementary domains is weak, so the limb bound to these non-alterable sites can dissociate from them. When the binding part between a limb and a site is more than two complementary domains, we assume the limb cannot leave the site by itself (the limb is trapped on that site). 


\subsubsection{Alterable sites and mechanisms.}

An alterable site has three features: (1) it can be in one of three states, "on", "off", or "trapped"; (2) it may or may not trap a spider; (3) it may contain a signal that can be sent out once a spider has attached to the site. In the designs of NOT gate and gate cascades, we use the switch mechanisms to implement a NOT gate and use the exit mechanism to implement the gate cascades. A mechanism consists of a set of neighboring alterable sites along the same direction. An alterable site is a DNA strand with different structures tethered to the surface of the lattice. In the definitions of different mechanisms, we use the same label to represent the sites that have the same functionality, but the DNA structures for these sites with the same label may be different in different mechanisms. For example, mechanisms $S W_{1 \rightarrow 0}, S W_{0 \rightarrow 1}$ and exit all contain sites $s_{r}^{I}$ and $s_{r}^{I I}$. These two sites have different DNA structures in different mechanisms.

Define mechanisms as:

$$
\begin{aligned}
S W_{1 \rightarrow 0} & =\left\{\left(s_{1 \rightarrow 0}, \mathbf{p}_{1}\right),\left(s_{r}^{I}, \mathbf{p}_{2}\right),\left(s_{r}^{I I}, \mathbf{p}_{3}\right)\right\} \\
S W_{0 \rightarrow 1} & =\left\{\left(s_{0 \rightarrow 1}, \mathbf{p}_{1}\right),\left(s_{r}^{I}, \mathbf{p}_{2}\right),\left(s_{r}^{I I}, \mathbf{p}_{3}\right)\right\} \\
\text { exit } & =\left\{\left(s_{t}, \mathbf{p}_{1}\right),\left(s_{r}^{I}, \mathbf{p}_{2}\right),\left(s_{r}^{I I}, \mathbf{p}_{3}\right)\right\}
\end{aligned}
$$

where $\left(s, \mathbf{p}_{i}\right)$ in a mechanism indicates the location of site $s$ is $\mathbf{p}_{i}$, and $\mathbf{p}_{i} \in \mathbb{Z}^{2}$ must satisfy

$$
\left|\mathbf{p}_{i}-\mathbf{p}_{i-1}\right|=1 \text {, where } i=2,3 \text {. }
$$

When a spider moves on site $s_{t}$, it becomes trapped. When a spider moves to a site $s_{1 \rightarrow 0}$ or $s_{0 \rightarrow 1}$, it becomes trapped and its value becomes 0 or 1 . When the spider goes on moving over the latter two sites in these mechanisms, site $s_{r}^{I}$ releases the limb trapped on the first site, and traps the spider; site $s_{r}^{I I}$ releases the limb trapped on the second site. Since the first site has different implementations, sites $s_{r}^{I}$ and $s_{r}^{I I}$ must have different implementations in different mechanisms even though their functionality is the same in three mechanisms.

Figure 15 shows the implementations of mechanism exit and its operating procedure. Figure 16 shows the implementations and operating procedure for $S W_{1 \rightarrow 0}$. Since 
$S W_{0 \rightarrow 1}$ follows a similar design to $S W_{1 \rightarrow 0}$, we only show the implementation for it in Figure 17.

In the designs of AND gate and OR gate, we use sites $s_{u}$ and $s_{p}$ to control the spiders' behavior at the crossroads in the AND gate and OR gate. Figure 18 shows

${ }_{525}$ how site $s_{u}$ sends a "turning-on" signal to unblock the site $s_{p}$ when a spider attaches to it.

\subsection{Possible Implementation II}

In the second implementation, only DNA strand displacement is used. We add an excess amount of external strands in the environment to aid the movement of molecular spiders when they move through the exit mechanism and switch mechanisms. Each spider has two legs and one arm. There are two types of arm. Arm of type 1 could only attach to the normal site $s_{1}$ and arm of type 0 could only attach to the normal site $s_{0}$. Implementations of spider leg and arms are shown in Figure 19 . We add one type of external strand containing two domains 4 and $b$ in this implementation. Figure 20 shows the implementation of the exit mechanism. Figure 21 and Figure 22 show the implementations of a switch mechanisms $S W_{1 \rightarrow 0}$ and $S W_{0 \rightarrow 1}$. Sites $s_{u}$ and $s_{p}$ used in the AND/OR gate implementation remain the same design as in the first implementation.

\section{Discussion and Conclusion}

Using an active multi-spider model with spider cooperation and localized signal transmission, we have implemented the basic logic gates (AND, OR, NOT). We have shown how to implement gate cascades, in which each upstream gate $G_{u}$ is connected to a downstream gate $G_{d}$ using the exit mechanism. We use $O(1)$ types of spiders and sites. To evaluate an $n$-variable Boolean function that is in 3-CNF with $m$ clauses, the evaluation time is $O(\log m)$ and the size of the circuit is $O(m)$. Therefore, our design supports scalable computation and ensures spatial locality.

Molecular circuits with spatial locality overcome the challenges of computation speed-up and sequence reuse in molecular computing in a well-mixed environment, but there are still other issues. Previous work on tethered circuits [11, 12] spatially 

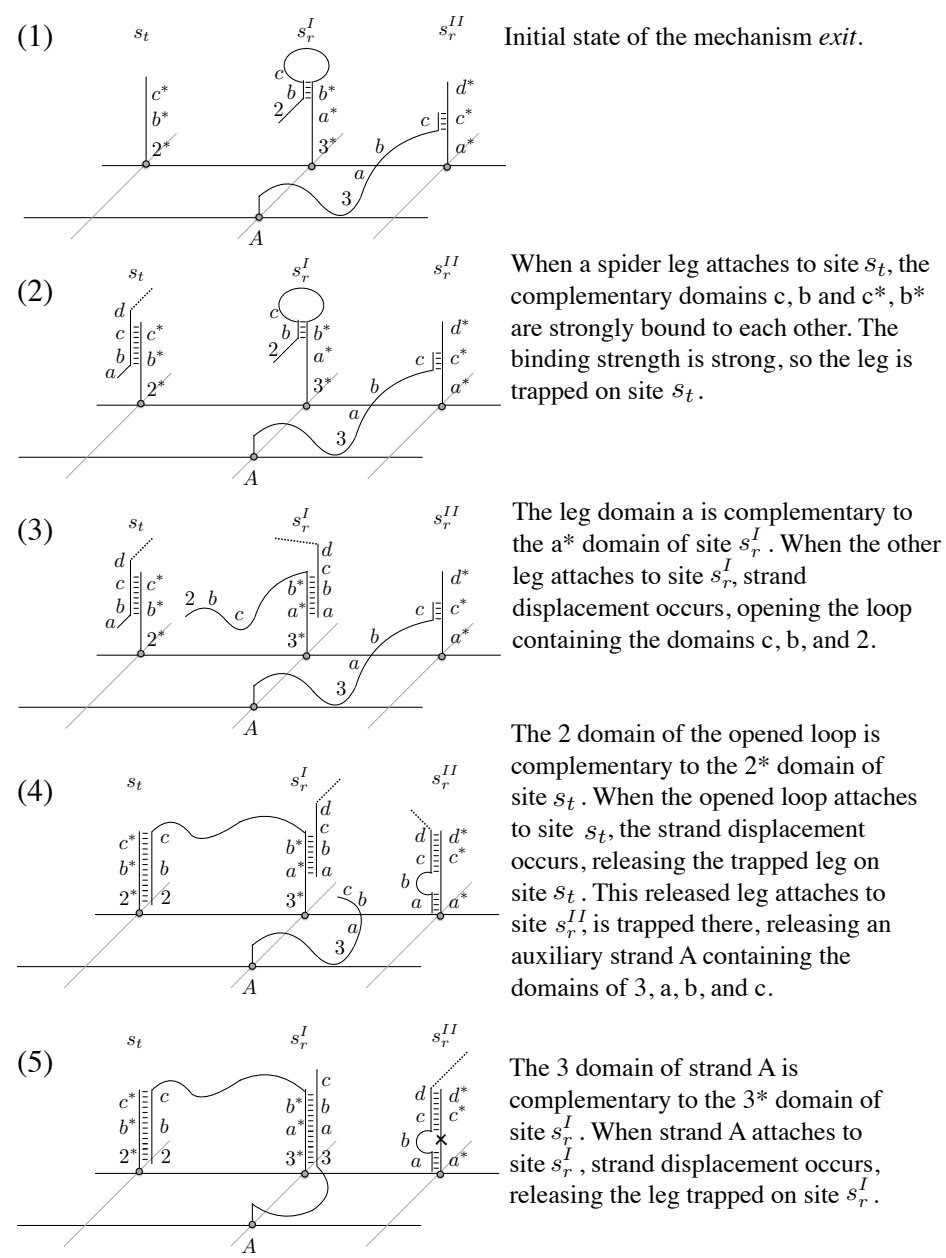

Figure 15: Possible implementation I. An implementation of mechanism exit. Diagram (1) is the initial condition of mechanism exit. Diagram (1) through (5) show the operating procedure of exit that cuts off the backward route of the spider. Each diagram has an explanation to the right of it. We omit the spider body in these diagrams. A dotted line of a limb represents the omitted part that extends to connect to the spider body. In diagram (5), the spider cannot move to sites $s_{t}$ and $s_{r}^{I}$ since no complementary domains exist between the leg and these sites. Therefore, the backward route of the spider is cut off when the spider moves through the exit mechanism. In diagram (5), the DNAzyme leg on site $s_{r}^{I I}$ cleaves the site at the position labeled with a small cross, making the spider move forward. 

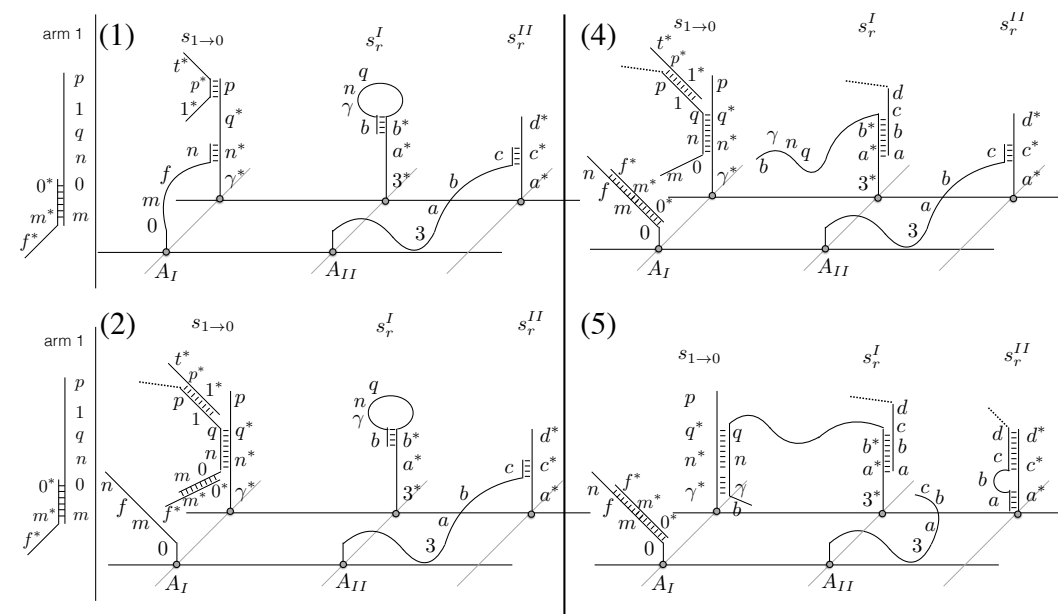

(5)
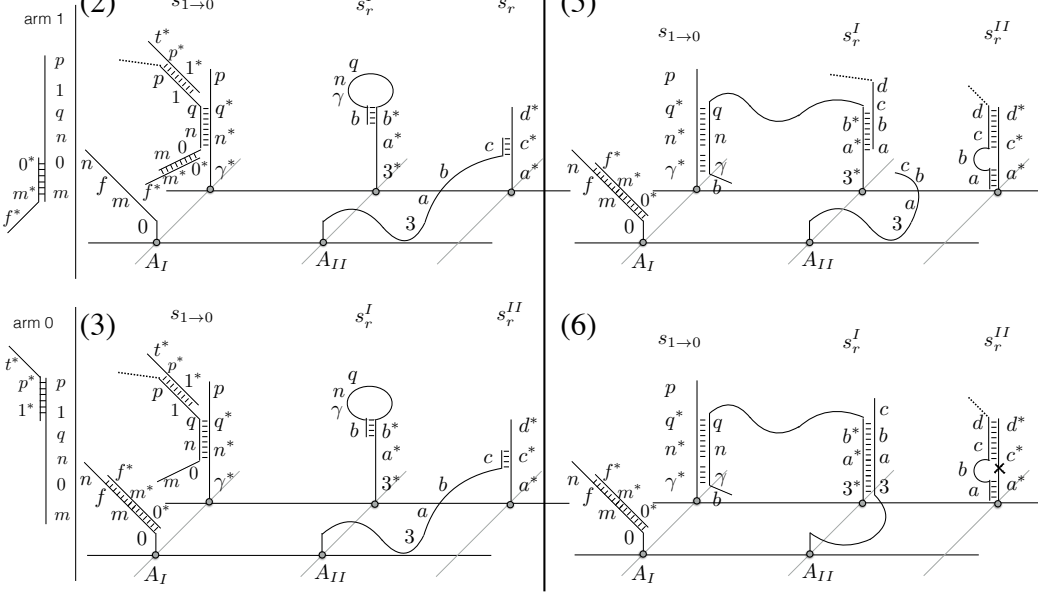

Figure 16: Possible implementation I. An implementation of the switch mechanism $S W_{1 \rightarrow 0}$. Diagram (1) shows the initial condition. When a spider with arm type of 1 , as shown to the left of diagram (1), reaches site $s_{1 \rightarrow 0}$, the arm type becomes 0 and the spider is trapped on that site. This procedure consists of two DNA strand displacements. Domain 1 of arm 1 binds to $1^{*}$ domain, the strand displacement occurs to take off the strand $t^{*} p^{*} 1^{*}$ from the site and binds it to the arm; Domain $q$ of arm 1 binds to $q^{*}$ domain, the strand displacement occurs to release the strand $A_{I}$ having domains of $n, f, m, 0$. The arm binds to the site via two complementary domains $\left(q, q^{*}\right.$ and $\left.n, n^{*}\right)$, the binding strength is high so the arm can not dissociate from the site by itself, as shown in diagram (2). Since the released strand $A_{I}$ contains an $f$ domain which can bind to the open end $f^{*}$ of the trapped arm, strand displacement occurs to take off the strand $f^{*} m^{*} 0^{*}$ from the arm, making the arm 0-typed, as shown in diagram (3). In diagram (4), one leg of the spider attaches to site $s_{r}^{I}$, the strand displacement opens the loop, and this leg is trapped. In diagram (5), the opened loop releases the arm trapped on $s_{1 \rightarrow 0}$, and the other leg attaches to site $s_{r}^{I I}$ releasing strand $A_{I I}$. In diagram (6), strand $A_{I I}$ displaces the leg trapped on site $s_{r}^{I}$ and the leg on site $s_{r}^{I I}$ can cleave the site at the position labeled with a small cross, thus the spider is free to move forward. Since the legs and the arm of the spider do not have any complementary domains to the current site $s_{1 \rightarrow 0}$ and $s_{r}^{I}$, the spider cannot move back. The arm type is 0 in diagram (4), (5), and (6). 


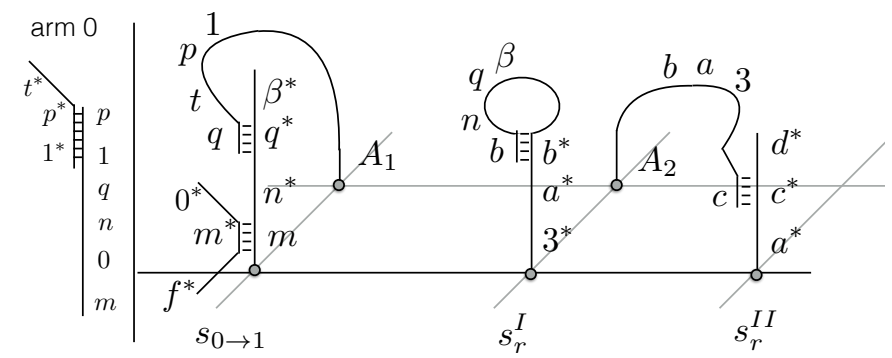

Figure 17: Possible implementation I. An implementation of the switch mechanism $S W_{0 \rightarrow 1}$. The operating procedure of this mechanism is similar to the one in $S W_{1 \rightarrow 0}$ shown in Figure 16 When arm 0 attaches to site $s_{0 \rightarrow 1}$, the strand displacement caused by the binding of $0,0^{*}$ domains takes off the strand $f^{*} m^{*} 0^{*}$ from the site and binds this strand to the arm, the strand displacement caused by the biding of $n, n^{*}$ domains releases the strand $A_{1}$. The released strand $A_{1}$ takes off the strand $t^{*} p^{*} 1^{*}$ from the arm, switching the type of the arm to 1 . The arm is trapped on $s_{0 \rightarrow 1}$, which could be released when one spider leg attaches to site $s_{r}^{I}$. When the arm is released, one spider leg is trapped on $s_{r}^{I}$. When the other leg of the spider attaches to site $s_{r}^{I I}$, strand $A_{2}$ is released, which can release the trapped leg on $s_{r}^{I}$.

isolates different gates on a surface, e.g., a DNA origami tile [27], such that only gates in close proximity can interact with each other, and two computation units that are not adjacent to each other can use the same sequence. However, the tethered circuits [11, 12] lack a direct implementation of the NOT gate, and circuit verification needs to verify many complicated reactions involved in the computation. Previous work [28] has used a walker system to construct logic circuits with spatial locality, but it lacks modularity and is limited to sequential evaluation due to its design where the circuit constructed is in the form of a Binary Decision Diagram (BDD). A walker initially placed at the root node walks along a path unblocked by externally-added strands to reach a leaf node representing True or False, causing a fluorophore change to report the computation result. For practical reasons, this reporting strategy needs two parallel circuits that detect fluorophore change at the True nodes and False nodes respectively to avoid ambiguity. Our design uses the reporting spider to avoid reporting problems [28], and we support parallel evaluation. As a result, to evaluate an $m$-clause 3-CNF circuit, we need time $O(\log m)$ while the circuit [28] needs time $O(m)$. We use the same linear space complexity $O(m)$ as in the circuit [28], and it is easier to construct large circuits using our design because of its modularity. Compared with previous work 

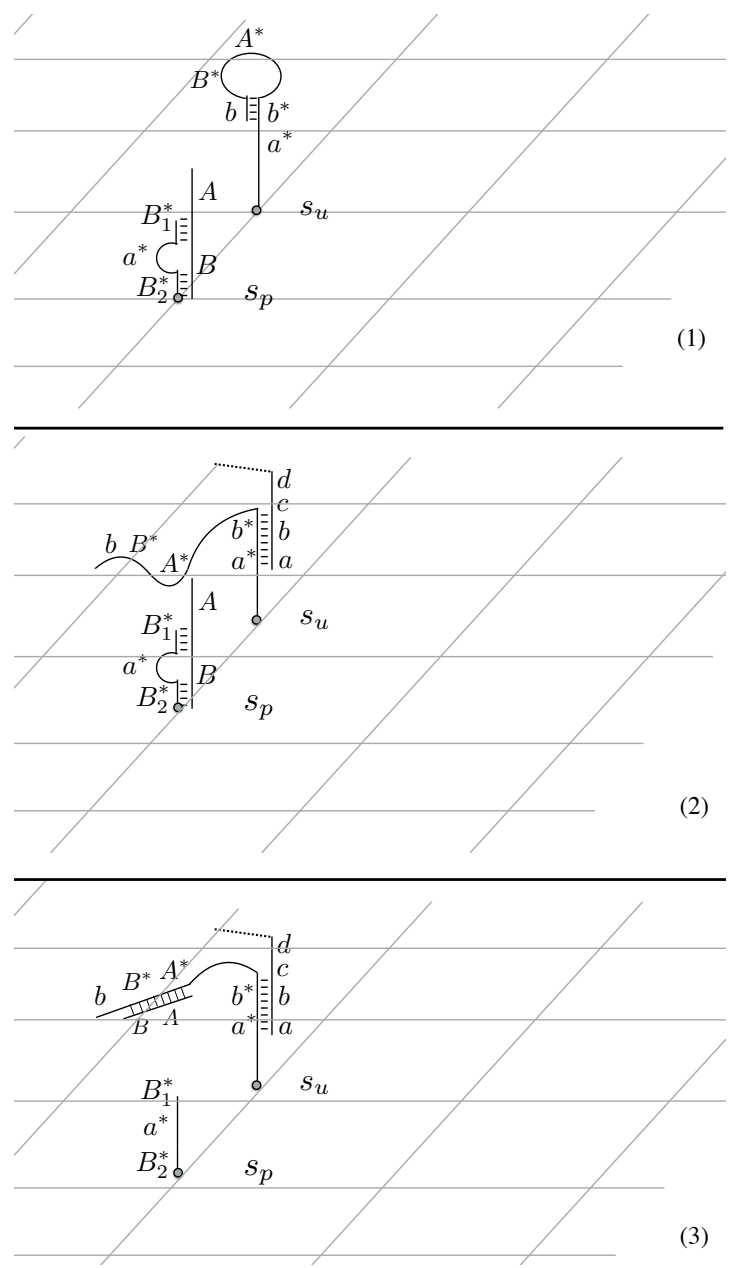

Figure 18: Possible implementation I. An implementation of sites $s_{u}$ and $s_{p}$ used in the AND gate and the OR gate. Diagram (1) shows the initial condition. Domain $a^{*}$ forms a small loop on site $s_{p}$, which is hard to bind to a spider limb, so a spider cannot attach to site $s_{p}$ initially. In diagram (2), when a spider leg attaches to site $s_{u}$, strand displacement occurs, opening the loop $A^{*} B^{*} b$. The binding parts between the spider leg and site $s_{u}$ are two pairs of complementary domains, so the binding strength is high enough to trap the spider leg. The opened loop $A^{*} B^{*} b$ has the complementary part $A$ on site $s_{p}$. In Diagram (3), strand displacement takes place when the opened loop binds to the $A$ domain on site $s_{p}$, which removes strand $A B$ from site $s_{p}$, thus domain $a^{*}$ becomes available for the spider limb. The state of site $s_{p}$ is turned on in diagram (3). 


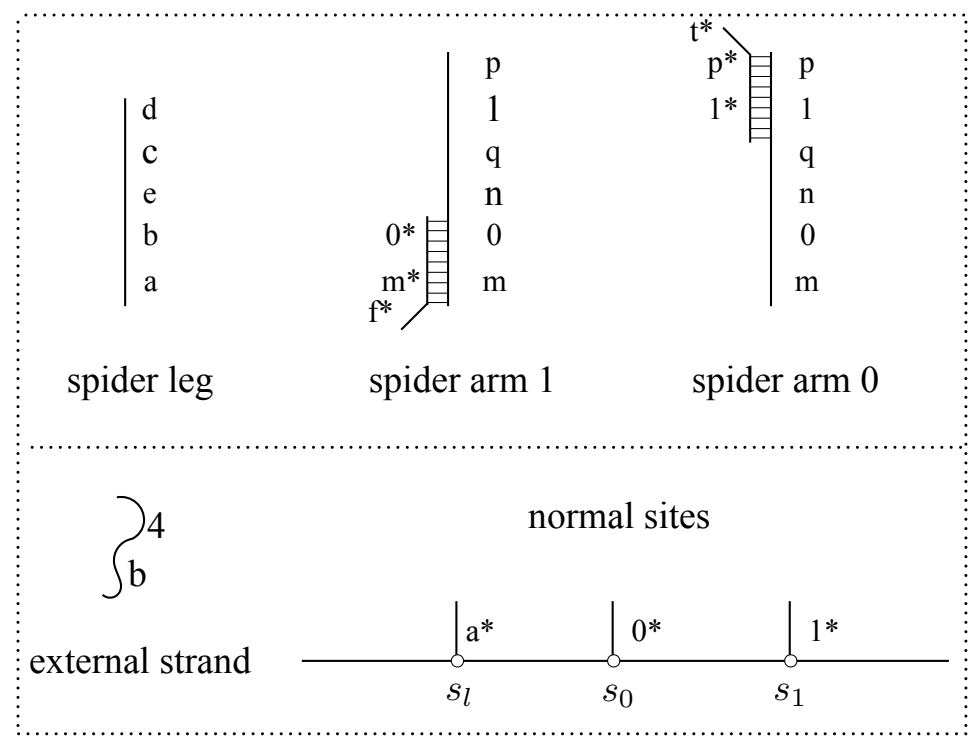

Figure 19: Possible implementation II. Implementations of spider limbs, external strands, and normal sites. A spider leg contains five domains. Normal sites keep the same structures as in the first implementation. An excess amount of external strands diffuse freely in the environment. External strands only participate in reactions of DNA strand displacement when a spider is attached to a functional site $s_{r}^{I I}$. 


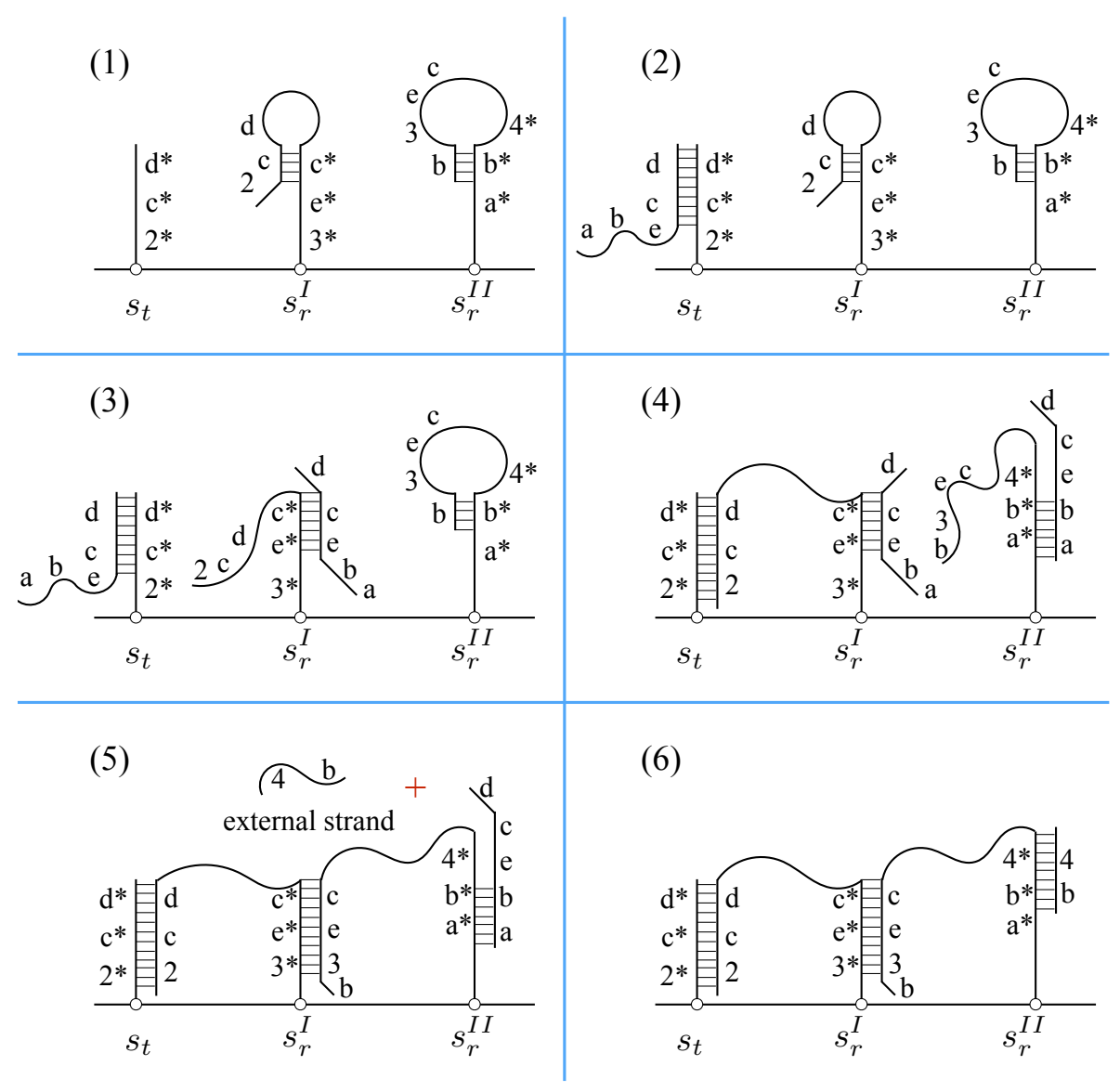

Figure 20: Possible implementation II. An implementation of mechanism exit using DNA strand displacement. Diagram (1) shows initial conditions. In Diagram (2), a spider leg attaches to site $s_{t}$ and is trapped on that site. In Diagram (3), when the other leg of the spider attaches to site $s_{r}^{I}$, strand displacement occurs to open the loop of site $s_{r}^{I}$. Spider is trapped on site $s_{r}^{I}$, and a signal containing domains $2, c$ and $d$ can reach the neighboring sites of site $s_{r}^{I}$. In diagram (4), the released signal containing domains 2,c and $d$ reacts with site $s_{t}$ to cause strand displacement, which frees the trapped leg on site $s_{t}$. The released leg attaches to site $s_{r}^{I I}$ and is trapped on site $s_{r}^{I I}$. Strand displacement occurs between the spider leg and site $s_{r}^{I I}$, domain $4^{*}$ becomes active because the loop is opened, and a signal containing domains $b, 3, e$ and $c$ is released. In diagram (5), the released signal containing domains $b, 3, e$ and $c$ reacts with site $s_{r}^{I}$ to free the trapped leg. Since domain $4^{*}$ is exposed, external strands containing domains $4, b$ can react with site $s_{r}^{I I}$, which would cause strand displacement. In diagram (6), the trapped leg on site $s_{r}^{I I}$ is released. Both legs of the spider are free to move forward, and the spider cannot move back because sites $s_{t}$ and $s_{r}^{I}$ are blocked. 
(1)
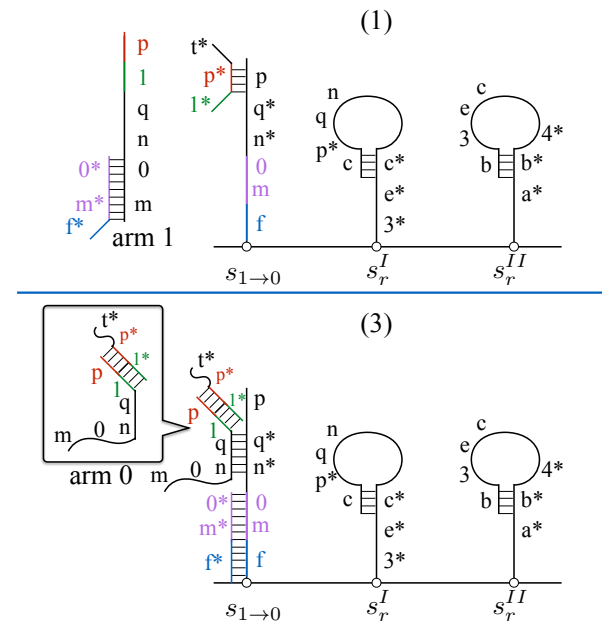

(5)

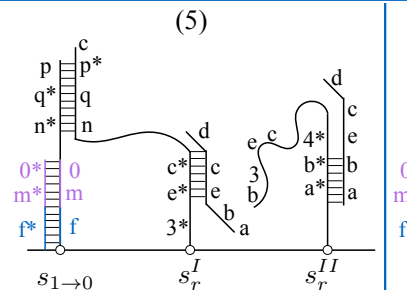

(3)

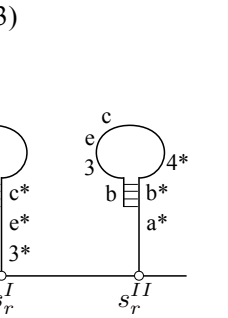

(2)

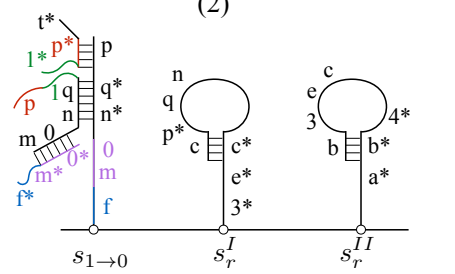

(4)

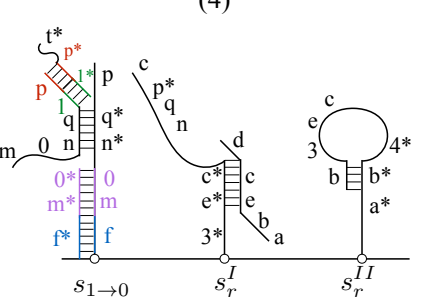

(7)

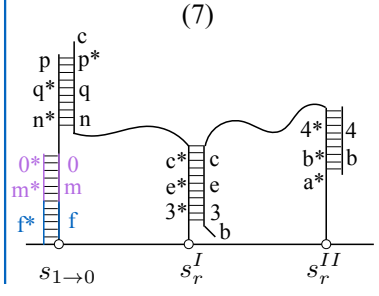

Figure 21: Possible implementation II. An implementation of switch mechanism $S W_{1 \rightarrow 0}$. Diagram (1) shows initial conditions. The arm of type 1 binds to site $s_{1 \rightarrow 0}$ via two complementary domains $\left(q, q^{*}\right.$ and $\left.n, n^{*}\right)$, and is trapped on site $s_{1 \rightarrow 0}$ because the binding strength is high, as shown in diagram (2). In diagram (3), two kinds of strand displacements occur between the trapped arm and site $s_{1 \rightarrow 0}$. Domains $p$ and 1 of the arm strip off the strand having domains $p^{*}, 1^{*}, t^{*}$ from site $s_{1 \rightarrow 0}$. Domains $0, m$ and $f$ of site $s_{1 \rightarrow 0}$ strip off the strand having domains $0^{*}, m f^{*}$ from the arm. The type of the arm becomes 0 after these two strand displacements. Diagrams (4) - (7) show similar reactions between sites $s_{r}^{I}, s_{r}^{I I}$ and spider as the reactions shown in the exit mechanism implementation (diagrams (3) - (6) in Figure 20,. When a spider leaves site $s_{r}^{I I}$, as shown in diagram (7), its backward route is cut off because sites $s_{r}^{I}$ and $s_{r}^{I I}$ are blocked. 


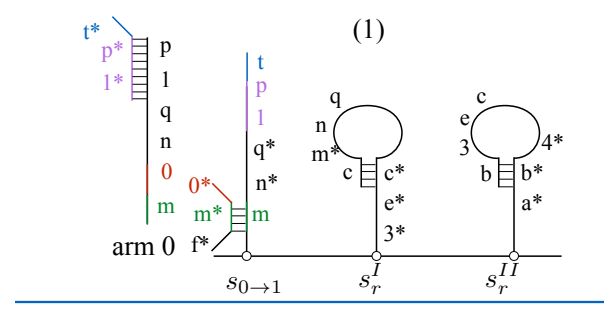

(3)
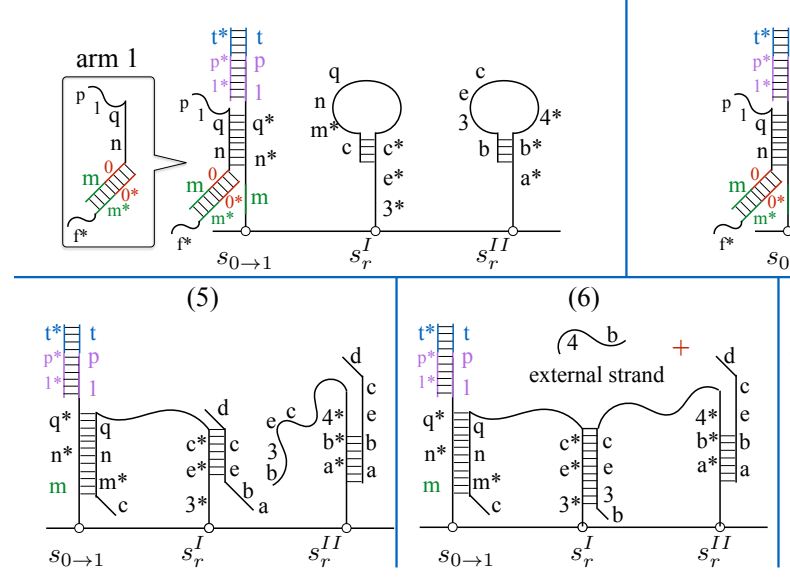

(2)

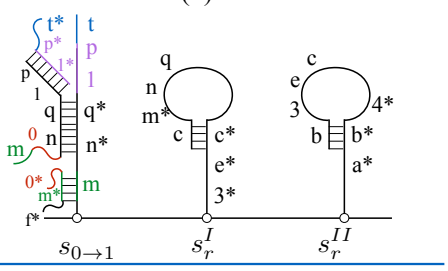

(4)

Figure 22: Possible implementation II. An implementation of switch mechanism $S W_{0 \rightarrow 1}$. Diagram (1) shows initial conditions. The arm type 0 binds to site $s_{0 \rightarrow 1}$ via two complementary domains $\left(q, q^{*}\right.$ and $\left.n, n^{*}\right)$, and is trapped on site $s_{0 \rightarrow 1}$, as shown in diagram (2). In diagram (3), two kinds of strand displacement occur between the trapped arm and site $s_{0 \rightarrow 1}$. Domains 0 and $m$ of the arm strip off the strand having domains $0^{*}, m^{*}, f^{*}$ from site $s_{0 \rightarrow 1}$. Domains $t, p, 1$ of site $s_{0 \rightarrow 1}$ strip off the strand having domains $t^{*}, p^{*}, 1^{*}$ from the arm. The type of the arm becomes 1 after these two strand displacements. Diagrams (4) - (7) are similar reactions as the procedures shown in the implementation of switch mechanism $S W_{1 \rightarrow 0}$ (diagrams (4) - (7) in Figure 21. When a spider leaves site $s_{r}^{I I}$, as shown in diagram (7), its backward route is cut off because sites $s_{r}^{I}$ and $s_{r}^{I I}$ are blocked. 
[11, 12, 28], our design better addresses the following issues:

Geometric layout. Molecular circuits with spatial locality arrange different computing components on a 2D plane where the distance between different components should be set carefully to avoid interference across components. Reducing the number of gates used in a circuit can ease the geometric layout problem. Our design implements a NOT gate to avoid dual-rail logic conversion used in previous work [11, 12], which simplifies the circuit and its layout. Compared with the circuit [28] in a form of BDD where the layout of different branching paths requires appropriate angles and lengths, our design only considers connections between gates because each gate has a fixed layout.

Data encoding. In previous work, variable representation is encoded into the circuit [11, 12, 28], so each variable corresponds to a distinct sequence. This complicates sequence design if the circuit has a large number of variables. Our design separates variable representation from circuit design, only using two types of spiders placed at different input locations to represent all variables.

Circuit reusability. Tethered circuits [11, 12] use irreversible local signal transmission to implement logic computation and value propagation, so the circuit is not reusable. The circuit [28] adds external strands to unblock a path for an evaluating walker. This procedure irreversibly changes the circuit configuration, thus the circuit is not reusable. In our design, irreversible local signal transmission is used to control the spiders' behavior at a few locations in the circuit, which only occupy a small portion of computation. Since non-alterable sites form the majority of the circuit, most parts of the circuit are reusable.

The performance of our circuit is influenced by the input assignments, the circuit size, and the circuit height (the height of a binary AST representing the circuit). For a large circuit, computation time would span a large range, which makes it hard to predict the overall circuit performance. We propose two methods to improve the circuit predictability in Section 9 


\section{Future Work}

595 lator that simulates the circuit at the site level, assuming spiders have equal transition rates to all reachable sites. We have sketched two kinds of implementations where normal sites are short DNA strands so that molecular spiders can attach to or detach from the normal sites freely, and functional sites transmit signals to neighboring sites via strand displacement. In the future, we will complete a plausible implementation and focus on a simulator that can better reflect how different sites react with spiders according to that implementation.

As circuit size increases, computation time variance increases, making it hard to predict the circuit performance. To improve the circuit predictability, we can simplify the Boolean expression of the circuit and reduce the height of the circuit tree, because Section 6 shows that the standard deviation of computation time increases as circuit size and circuit height increase. Another method to improve the circuit predictability is to make the spider move unidirectionally. Computation time distribution would be more concentrated in that new model, and the circuit predictability improved.

In circuit verification, we do not consider the failures of mechanisms that are implemented using the hairpin structures in Section7. The loop of a hairpin structure may be opened spontaneously without interacting with other strands. If the signal encoded in the loop is thus released, it may cause unintended changes to the environment, which may cause errors in computation (spider with the wrong value reaches the output location) or lead to incomplete computation (walking paths for spiders are blocked). In the

We lack an experimental implementation of our designs, thus here we use a simufuture, we will explore circuit robustness by incorporating the failures of the mechanisms into our model.

\section{Acknowledgments}

This material is based upon work supported by the National Science Foundation under grants 1318833 and 1422840. 
[1] S. F. Wickham, J. Bath, Y. Katsuda, M. Endo, K. Hidaka, H. Sugiyama, A. J. Turberfield, A DNA-based molecular motor that can navigate a network of tracks, Nature Nanotechnology 7 (3) (2012) 169-173.

[2] K. Lund, A. J. Manzo, N. Dabby, N. Michelotti, A. Johnson-Buck, J. Nangreave, S. Taylor, R. Pei, M. N. Stojanovic, N. G. Walter, et al., Molecular robots guided by prescriptive landscapes, Nature 465 (7295) (2010) 206-210.

[3] O. Semenov, M. J. Olah, D. Stefanovic, Mechanism of diffusive transport in molecular spider models, Physical Review E 83 (2) (2011) 021117.

[4] L. Samii, G. A. Blab, E. H. C. Bromley, H. Linke, P. M. G. Curmi, M. J. Zuckermann, N. R. Forde, Time-dependent motor properties of multipedal molecular spiders, Physical Review E 84 (2011) 031111.

[5] M. Rank, L. Reese, E. Frey, Cooperative effects enhance the transport properties of molecular spider teams, Physical Review E 87 (2013) 032706.

[6] M. J. Olah, D. Stefanovic, Superdiffusive transport by multivalent molecular walkers moving under load, Phys. Rev. E 87 (2013) 062713.

[7] T. Omabegho, R. Sha, N. C. Seeman, A bipedal DNA brownian motor with coordinated legs, Science 324 (5923) (2009) 67-71.

[8] H. Gu, J. Chao, S.-J. Xiao, N. C. Seeman, A proximity-based programmable DNA nanoscale assembly line, Nature 465 (7295) (2010) 202-205.

[9] W. B. Sherman, N. C. Seeman, A precisely controlled DNA biped walking device, Nano Letters 4 (7) (2004) 1203-1207.

[10] J.-S. Shin, N. A. Pierce, A synthetic DNA walker for molecular transport, Journal of the American Chemical Society 126 (35) (2004) 10834-10835.

[11] R. A. Muscat, K. Strauss, L. Ceze, G. Seelig, DNA-based molecular architecture with spatially localized components, in: Proceedings of the 40th Annual International Conference on Computer Architecture (ISCA), ACM, 2013, pp. 177-188. 
[12] H. Chandran, N. Gopalkrishnan, A. Phillips, J. Reif, Localized hybridization circuits, in: DNA Computing and Molecular Programming, Springer, 2011, pp. 6483.

[13] R. Pei, S. K. Taylor, D. Stefanovic, S. Rudchenko, T. E. Mitchell, M. N. Stojanovic, Behavior of polycatalytic assemblies in a substrate-displaying matrix, Journal of the American Chemical Society 128 (39) (2006) 12693-12699.

[14] T. Antal, P. L. Krapivsky, Molecular spiders with memory, Physical Review E 76 (2) (2007) 021121.

[15] O. Semenov, M. J. Olah, D. Stefanovic, Multiple molecular spiders with a single localized source-the one-dimensional case, in: DNA Computing and Molecular Programming, Springer, 2011, pp. 204-216.

[16] M. J. Olah, D. Stefanovic, Multivalent random walkers - a model for deoxyribozyme walkers, in: DNA Computing and Molecular Programming, Springer, 2011, pp. 160-174.

[17] O. Semenov, D. Mohr, D. Stefanovic, First-passage properties of molecular spiders, Physical Review E 88 (1) (2013) 012724.

[18] J. E. Padilla, W. Liu, N. C. Seeman, Hierarchical self assembly of patterns from the Robinson tilings: DNA tile design in an enhanced tile assembly model, Natural Computing 11 (2) (2012) 323-338.

[19] L. Qian, E. Winfree, Scaling up digital circuit computation with DNA strand displacement cascades, Science 332 (6034) (2011) 1196-1201.

[20] M. R. Lakin, D. Stefanovic, Supervised learning in an adaptive DNA strand displacement circuit, in: DNA Computing and Molecular Programming, Springer, 2015, pp. 154-167.

[21] G. Seelig, D. Soloveichik, D. Y. Zhang, E. Winfree, Enzyme-free nucleic acid logic circuits, science 314 (5805) (2006) 1585-1588. 
[22] M. N. Stojanovic, T. E. Mitchell, D. Stefanovic, Deoxyribozyme-based logic gates, Journal of the American Chemical Society 124 (14) (2002) 3555-3561.

[23] R. Pei, S. K. Taylor, Deoxyribozyme-based autonomous molecular spiders controlled by computing logic gates, IPCBEE Proceedings.

[24] C. W. Brown, M. R. Lakin, D. Stefanovic, S. W. Graves, Catalytic molecular logic devices by DNAzyme displacement, ChemBioChem 15 (7) (2014) 950-954.

[25] H. Lederman, J. Macdonald, D. Stefanovic, M. N. Stojanovic, Deoxyribozymebased three-input logic gates and construction of a molecular full adder, Biochemistry 45 (4) (2006) 1194-1199.

[26] M. N. Stojanovic, D. Stefanovic, S. Rudchenko, Exercises in molecular computing, Accounts of Chemical Research 47 (6) (2014) 1845-1852.

[27] P. W. Rothemund, Folding DNA to create nanoscale shapes and patterns, Nature 440 (7082) (2006) 297-302.

[28] F. Dannenberg, M. Kwiatkowska, C. Thachuk, A. Turberfield, DNA walker circuits: Computational potential, design, and verification, in: D. Soloveichik, B. Yurke (Eds.), DNA Computing and Molecular Programming, Vol. 8141 of Lecture Notes in Computer Science, Springer International Publishing, 2013, pp. $31-45$. 